\title{
Stationary distribution and density function expression for a stochastic SIQRS epidemic model with temporary immunity
}

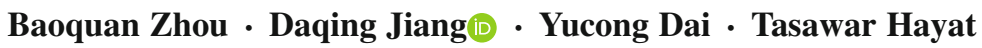

Received: 3 September 2020 / Accepted: 10 December 2020 / Published online: 8 June 2021

(C) The Author(s), under exclusive licence to Springer Nature B.V. 2021

\begin{abstract}
Recently, considering the temporary immunity of individuals who have recovered from certain infectious diseases, Liu et al. (Phys A Stat Mech Appl 551:124152, 2020) proposed and studied a stochastic susceptible-infected-recovered-susceptible model with logistic growth. For a more realistic situation, the effects of quarantine strategies and stochasticity should be taken into account. Hence, our paper focuses on a stochastic susceptible-infected-quarantined-recoveredsusceptible epidemic model with temporary immunity. First, by means of the Khas'minskii theory and Lyapunov function approach, we construct a critical value $\mathscr{R}_{0}^{S}$ corresponding to the basic reproduction number $\mathscr{R}_{0}$ of the deterministic system. Moreover, we prove that there is a unique ergodic stationary distribution if $\mathscr{R}_{0}^{S}>$ 1. Focusing on the results of Zhou et al. (Chaos Soliton Fractals 137:109865, 2020), we develop some suitable solving theories for the general four-dimensional Fokker-Planck equation. The key aim of the present study is to obtain the explicit density function expres-
\end{abstract}

D. Jiang $(\varangle) \cdot$ B. Zhou · Y. Dai

College of Science, China University of Petroleum (East China), Qingdao 266580, People's Republic of China e-mail: daqingjiang2010@ hotmail.com

D. Jiang · T. Hayat

Nonlinear Analysis and Applied

Mathematics(NAAM)-Research Group, Department of

Mathematics, King Abdulaziz University, Jeddah, Saudi

Arabia

T. Hayat

Department of Mathematics, Quaid-i-Azam University 45320, Islamabad 44000, Pakistan sion of the stationary distribution under $\mathscr{R}_{0}^{S}>1$. It should be noted that the existence of an ergodic stationary distribution together with the unique exact probability density function can reveal all the dynamical properties of disease persistence in both epidemiological and statistical aspects. Next, some numerical simulations together with parameter analyses are shown to support our theoretical results. Last, through comparison with other articles, results are discussed and the main conclusions are highlighted.

Keywords Stochastic SIQRS epidemic model . Temporary immunity · Ergodic stationary distribution . Fokker-Planck equation · Density function

\section{Introduction}

Over time, an increasing number of people are becoming concerned with health and the desire to improve the quality of life worldwide. However, major infectious diseases such as Ebola, avian influenza, cholera, and heptitis $\mathrm{B}$ are one of the biggest threats to public health [1-3]. Epidemiology, greatly supported by various mathematical models, is the study of the spread of diseases and trace factors that give rise to their occurrence. Following the classical SusceptibleInfected-Recovered (SIR) epidemic models proposed by Kermack and McKendrick [4], some authors have developed a series of reasonable ordinary differential equations (ODEs) to describe the transmission 
of various epidemics [5-13]. In [5], Liu et al. established an Susceptible-Vaccinated-Infected-Recovered (SVIR) epidemic model with vaccination strategies and obtained the corresponding global stability of equilibria. Hove-Musekwa and Nyabadza [9] considered a deterministic HIV/AIDS model taking into account the active screening of disease carriers and seeking of treatment. They also derived the relevant basic reproduction number. Considering the sequence diversity and highly infectious nature of some contagious diseases, we occasionally need to implement quarantine strategies to control the spread of disease. For example, without an effective vaccine, the rapid spread of coronavirus disease 2019 (COVID-19) worldwide has had a serious socioeconomic impact and imposed a potentially great threat to human safety [14]. Hence, many suitable SIQR epidemic models have been developed in the past few decades [15-19]. In [15], Herbert and Ma obtained the corresponding basic reproduction number of a deterministic SIQR model with quarantineadjusted incidence. Nevertheless, recovered individuals with temporary immunity may be susceptible to the disease again in the future [20-23]. Zhang et al. [20] studied the global asymptotic stability of two equilibria to a SIQS epidemic model with the nonlinear incidence rate $\frac{\beta S I}{f(I)}$. Following the above analyses, in this study, a deterministic SIQRS with temporary immunity is developed for further epidemiological investigation.

In our daily life, it is obvious that the spread of infection, travel of populations and the design of control strategies are critically perturbed by some environmental variations [24]. For dynamical study and simulation, by taking the effect of stochastic perturbation into consideration, some scholars considered and analyzed various stochastic differential equations (SDEs) for the spread of epidemics [25-37]. From [25], Zhao and Jiang established a universal theory of extinction and persistence in mean based on a stochastic SIS epidemic model with vaccination. Han and Jiang [27] introduced a stochastic staged progression AIDS model with second-order perturbation and proved the ergodicity of the global positive solution if $\mathcal{R}_{0}^{H}>1$. Considering the delay influence, Caraballo and Fatini [29] derived the existence of stationary distribution for a stochastic SIRS epidemic model with distributed delay. For cholera epidemic, a stochastic SIQRB infectious disease model was researched by Liu and Jiang [32]. Recently, Zhou and Zhang (2020) obtained the explicit expression of the probability density function for the three-dimensional avian-only influenza model, which is described in [33].

Focusing on the temporary immunity phenomenon of infected people, quarantine strategies and random perturbations, our study aims to develop a stochastic SIQRS epidemic system with temporary immunity. As is well known, the corresponding basic reproduction number and unique endemic equilibrium can reflect the disease persistence of a deterministic system. Nevertheless, the positive equilibrium no longer exists in a stochastic model owing to the effect of unpredictable environmental noises. Hence, ergodicity theory and the existence of stationary distribution, which greatly reflect the stochastic permanence of disease, are gradually becoming more popular in the transmission of epidemics. In practical application, some statistical properties of epidemic models still need to be estimated to effectively prevent and control the spread of infectious diseases. Notably, there are relatively few studies devoted to deriving the explicit expression of probability density function due to the difficulty of solving the high-dimensional Fokker-Planck equation. To the best of our knowledge, some studies of probability density functions for stationary distributions are shown in the present study. As a result, we will concentrate on the following three aims: (i) construct a reasonable stochastic threshold $\mathscr{R}_{0}^{S}$ corresponding to the basic reproduction number $\mathscr{R}_{0}$; (ii) investigate the disease persistence of stochastic SIQRS model under $\mathscr{R}_{0}^{S}>1$, namely, the existence of the uniqueness of an ergodic stationary distribution and the exact expression of this unique probability density function; and (iii) provide the corresponding numerical simulations and parameter analyses for our analytical results.

The rest of our study is arranged as follows. For the later dynamical investigation, Sect. 2 introduces the corresponding mathematical models, important notations and necessary lemmas. By constructing a series of suitable Lyapunov functions, a stochastic critical value $\mathscr{R}_{0}^{S}$ involved in the random noises is obtained. Based on the global positive solution property and Khas'minskii theory, Sect. 3 shows that there is a unique ergodic stationary distribution when $\mathscr{R}_{0}^{S}>1$. In Sect. 4, by developing some solving theories of the relevant algebraic equations, the corresponding fourdimensional Fokker-Planck equation is solved for the explicit expression of log-normal density function to the stochastic model if $\mathscr{R}_{0}^{S}>1$. Section 5 presents some empirical examples and parameter analyses to 
validate the above theoretical results. Finally, the relevant results are discussed and the main conclusions are introduced by comparison with the existing results in Sect. 6.

\section{Mathematical models and necessary lemmas}

\subsection{Deterministic SIQRS epidemic model and dynamical properties}

Given the above descriptions, we assume that the investigated population $N(t)$ can be divided into susceptible $S(t)$, infectious $I(t)$, quarantined $Q(t)$ and recovered $R(t)$ individuals at time $t$. A deterministic SIQRS epidemic model with temporary (short-term) immunity is studied herein, which is given by

$$
\left\{\begin{array}{l}
\frac{\mathrm{d} S(t)}{\mathrm{d} t}=\Lambda-\mu S(t)-\beta S(t) I(t)+\omega R(t), \\
\frac{\mathrm{d} I(t)}{\mathrm{d} t}=\beta S(t) I(t)-\left(\mu+\alpha_{1}+\delta+\gamma\right) I(t), \\
\frac{\mathrm{d} Q(t)}{\mathrm{d} t}=\delta I(t)-\left(\mu+\alpha_{2}+\varepsilon\right) Q(t), \\
\frac{\mathrm{d} R(t)}{\mathrm{d} t}=\gamma I(t)+\varepsilon Q(t)-(\mu+\omega) R(t),
\end{array}\right.
$$

where $\Lambda$ is the recruitment rate of the susceptible individuals, $\mu$ depicts the natural death rate of the population, $\beta$ is the transmission rate, $\alpha_{1}, \alpha_{2}$ represent the average disease-induced death rate of the infected and quarantined individuals, respectively, $\delta$ denotes the isolated rate of the infected individuals, $\gamma$ and $\varepsilon$ are the recovery rate of the infected and quarantined individuals, and $\omega$ denotes the immune loss rate of the recovered individuals. All the parameters are assumed to be positive constants.

In the similar methods described by Ma and Zhou [21], system (2.1) has the corresponding basic reproduction number and the invariant attracting set, which are given by

$$
\begin{aligned}
\mathscr{R}_{0} & =\frac{\Lambda \beta}{\mu\left(\mu+\alpha_{1}+\delta+\gamma\right)}, \\
\Theta= & \{(S, I, Q, R) \mid S \geq 0, I \geq 0, Q \geq 0, R \geq 0, \\
& \left.S+I+Q+R \leq \frac{\Lambda}{\mu}\right\} .
\end{aligned}
$$

Additionally, two possible equilibria are shown as follows. (i) The disease-free equilibrium $E_{0}=\left(S_{0}\right.$,
$\left.I_{0}, Q_{0}, R_{0}\right)=\left(\frac{\Lambda}{\mu}, 0,0,0\right)$. (ii) The endemic equilibrium $E^{*}=\left(S^{*}, I^{*}, Q^{*}, R^{*}\right)=\left(\frac{\Lambda}{\mu \mathcal{R}_{0}}, \frac{\Lambda\left(\mathscr{R}_{0}-1\right)}{\varrho_{1} \mathscr{R}_{0}}\right.$, $\left.\frac{\Lambda \delta\left(\mathscr{R}_{0}-1\right)}{\varrho_{1}\left(\mu+\alpha_{2}+\varepsilon\right) \mathscr{R}_{0}}, \frac{\Lambda \varrho_{2}\left(\mathscr{R}_{0}-1\right)}{(\mu+\omega)\left(\mu+\alpha_{2}+\varepsilon\right) \mathscr{R}_{0}}\right)$, where $\varrho_{1}=\mu+$ $\alpha_{1}+\frac{\mu\left[(\gamma+\delta)\left(\mu+\alpha_{1}+\varepsilon\right)+\omega\left(\mu+\alpha_{2}\right)\right]}{(\mu+\omega)\left(\mu+\alpha_{1}+\varepsilon\right)}>0, \varrho_{2}=\gamma\left(\mu+\alpha_{2}+\right.$ $\varepsilon)+\varepsilon \delta>0$. These two equilibria have the following dynamical properties.

- If $\mathscr{R}_{0} \leq 1$, then $E_{0}$ is globally asymptotically stable in $\Theta$, which means the disease will be eradicated in a population.

- If $\mathscr{R}_{0}>1$, then $E^{*}$ is globally asymptotically stable, but $E_{0}$ is unstable in the domain $\Theta$. This indicates the disease will prevail and persist long-term.

\subsection{Stochastic SIQRS epidemic system}

In reality, the dynamical behavior of most epidemics is inevitably affected by random factors in nature. By means of the relevant assumptions and forms of stochastic perturbations developed in [25-30,32-34], in this study, we assume that these stochastic noises are directly proportional to the groups $S(t), I(t), Q(t)$ and $R(t)$. Hence, the corresponding stochastic SIQRS epidemic model with temporary immunity is described by

$$
\left\{\begin{aligned}
\mathrm{d} S(t)= & {[\Lambda-\mu S(t)-\beta S(t) I(t)} \\
& +\omega R(t)] \mathrm{d} t+\sigma_{1} S(t) d B_{1}(t), \\
\mathrm{d} I(t)= & {\left[\beta S(t) I(t)-\left(\mu+\alpha_{1}+\delta+\gamma\right) I(t)\right] \mathrm{d} t } \\
& +\sigma_{2} I(t) d B_{2}(t), \\
\mathrm{d} Q(t)= & {\left[\delta I(t)-\left(\mu+\alpha_{2}+\varepsilon\right) Q(t)\right] \mathrm{d} t } \\
& +\sigma_{3} Q(t) d B_{3}(t), \\
\mathrm{d} R(t)= & {[\gamma I(t)+\varepsilon Q(t)-(\mu+\omega) R(t)] \mathrm{d} t } \\
& +\sigma_{4} R(t) d B_{4}(t),
\end{aligned}\right.
$$

where $B_{1}(t), B_{2}(t), B_{3}(t)$ and $B_{4}(t)$ are four independent standard Brownian motions, and $\sigma_{i}^{2}>0(i=$ $1,2,3,4)$, respectively, denote their intensities.

\subsection{Mathematical notations and necessary lemmas}

Throughout this study, unless otherwise specified, let $\left\{\Omega, \mathscr{F},\left\{\mathscr{F}_{t}\right\}_{t \geq 0}, \mathbb{P}\right\}$ be a complete probability space with a filtration $\left\{\mathscr{F}_{t}\right\}_{t \geq 0}$ satisfying the usual conditions (i.e., it is increasing and right continuous while $\mathscr{F}_{0}$ contains all $\mathbb{P}$-null sets). For detailed descriptions, refer 
to Mao [36]. Moreover, for convenience and simplicity, all stochastic approaches and theories are based on the above space.

In order to study the later dynamical behavior of the stochastic system (2.2), some common notations shall be defined in the first place. Let $\mathbb{R}^{n}$ be an $n$-dimensional Euclidean space and

$$
\begin{aligned}
& \mathbb{R}_{+}^{k}=\left\{\left(x_{1}, \ldots, x_{k}\right) \mid x_{i}>0,1 \leq i \leq k\right\}, \\
& \mathbb{U}_{k, 4}=\left(\frac{1}{k}, k\right) \times\left(\frac{1}{k}, k\right) \times\left(\frac{1}{k}, k\right) \times\left(\frac{1}{k}, k\right) .
\end{aligned}
$$

In addition, let $A^{\tau}$ be the transposed matrix of the inverse matrix $A$, and $A^{-1}$ be the relevant inverse matrix of $A$.

Next, the corresponding global existence of the positive solution to the system (2.2) is introduced as follows.

Lemma 2.1 For any initial value $(S(0), I(0), Q(0)$, $R(0)) \in \mathbb{R}_{+}^{4}$, there is a unique solution $(S(t)$, $I(t), Q(t), R(t))$ of the system (2.2) on $t \geq 0$, and the solution will remain in $\mathbb{R}_{+}^{4}$ with probability one (a.s.).

The detailed proof of Lemma 2.1 is mostly similar to that in Theorem 3.1 of Liu and Jiang [37], so we omit it here.

By means of the Khas'minskii theory [38], considering the following stochastic differential equation defined in the space $\mathbb{R}^{n}$,

$d X(t)=\psi(X(t)) d t+\sum_{k=1}^{n} \sigma_{k}(X) d B_{k}(t)$,

where the diffusion matrix $\mathcal{F}(X)=\left(\bar{a}_{i j}(X)\right)$, and $\bar{a}_{i j}(X)=\sum_{k=1}^{n} \sigma_{k}^{i}(X) \sigma_{k}^{j}(X)$. Furthermore, the relevant existence theory of the unique ergodic stationary distribution is shown by the following Lemma 2.2.

Lemma 2.2 (Khas'minskii [38]) The Markov process $X(t)$ has a unique ergodic stationary distribution $\varpi(\cdot)$ if there exists a bounded domain $\mathbb{D} \subset \mathbb{R}^{n}$ with a regular boundary $\Gamma$ and

$\left(\mathscr{A}_{1}\right)$. There is a positive number $\kappa_{0}$ such that $\sum_{i, j=1}^{n} \bar{a}_{i j}(x) \xi_{i} \xi_{j} \geq \kappa_{0}|\xi|^{2}$ for any $x \in \mathbb{D}, \xi \in \mathbb{R}^{n}$.

$\left(\mathscr{A}_{2}\right)$. There is a non-negative $C^{2}$-function $V(x)$ such that $\mathscr{L} V(x)$ is negative for any $x \in \mathbb{R}^{n} \backslash \mathbb{D}$.

Then for all $x \in \mathbb{R}^{n}$ and integral function $\phi(\cdot)$ with respect to the measure $\phi(\cdot)$, it follows that

$\mathbb{P}\left\{\lim _{t \rightarrow \infty} \frac{1}{t} \int_{0}^{t} \phi(X(s)) d s=\int_{\mathbb{R}^{n}} \phi(x) \varpi(d x)\right\}=1$.
Now, by the relevant definitions described in Zhou and Zhang [33], we will develop some solving theories for the corresponding four-dimensional algebraic equations, which are described by the following Lemmas 2.3-2.5.

Lemma 2.3 Let $\theta_{0}$ be a symmetric matrix in the fourdimensional algebraic equation $G_{0}^{2}+A_{0} \theta_{0}+\theta_{0} A_{0}^{\tau}=0$, where $G_{0}=\operatorname{diag}(1,0,0,0)$, and

$A_{0}=\left(\begin{array}{cccc}-a_{1} & -a_{2} & -a_{3} & -a_{4} \\ 1 & 0 & 0 & 0 \\ 0 & 1 & 0 & 0 \\ 0 & 0 & 1 & 0\end{array}\right)$

Assuming that $a_{1}>0, a_{3}>0, a_{4}>0$, and $a_{1}\left(a_{2} a_{3}-\right.$ $\left.a_{1} a_{4}\right)-a_{3}^{2}>0$, then $\theta_{0}$ is a positive definite matrix.

Lemma 2.4 Let $\theta_{1}$ be a symmetric matrix in the fourdimensional algebraic equation $G_{0}^{2}+B_{0} \theta_{1}+\theta_{1} B_{0}^{\tau}=0$, where $G_{0}=\operatorname{diag}(1,0,0,0)$, and

$B_{0}=\left(\begin{array}{cccc}-b_{1} & -b_{2} & -b_{3} & -b_{4} \\ 1 & 0 & 0 & 0 \\ 0 & 1 & 0 & 0 \\ 0 & 0 & 1 & b_{44}\end{array}\right)$

If $b_{1}>0, b_{3}>0$, and $b_{1} b_{2}-b_{3}>0$, then $\theta_{1}$ is semi-positive definite.

Lemma 2.5 Let $\theta_{2}$ be a symmetric matrix in the fourdimensional algebraic equation $G_{0}^{2}+C_{0} \theta_{2}+\theta_{2} C_{0}^{\tau}=0$, where $G_{0}=\operatorname{diag}(1,0,0,0)$, and

$C_{0}=\left(\begin{array}{cccc}-c_{1} & -c_{2} & -c_{3} & -c_{4} \\ 1 & 0 & 0 & 0 \\ 0 & 0 & c_{33} & c_{34} \\ 0 & 0 & c_{43} & c_{44}\end{array}\right)$

If $c_{1}>0$ and $c_{2}>0$, then $\theta_{2}$ is semi-positive definite.

Remark 2.6 For convenience, $A_{0}, B_{0}$ and $C_{0}$ are, respectively, called standard $R_{1}, R_{2}, R_{3}$ matrices in this study. The corresponding proofs of Lemmas 2.3-2.5 are separately shown in subsections (I), (II) and (III) of "Appendix A". 


\section{Stationary distribution and ergodicity of system}

In this section, by Lemmas 2.1 and 2.2, we are devoted to obtain the sufficient conditions for ergodicity of the global positive solution and the existence of stationary distribution. First, we define

$\mathscr{R}_{0}^{S}=\frac{\Lambda \beta}{\left(\mu+\frac{\sigma_{1}^{2}}{2}\right)\left(\mu+\alpha_{1}+\gamma+\delta+\frac{\sigma_{2}^{2}}{2}\right)}$.

Theorem 3.1 Assuming that $\mathscr{R}_{0}^{S}>1$, for any initial value $(S(0), I(0), Q(0), R(0)) \in \mathbb{R}_{+}^{4}$, then the solution ( $S(t), I(t), Q(t), R(t))$ of the system (2.2) is ergodic and has a unique stationary distribution $\varpi(\cdot)$.

Proof By Lemma 2.1, we derive that there is a unique global positive solution $(S(t), I(t), Q(t), R(t)) \in \mathbb{R}_{+}^{4}$. Hence, the proof of Theorem 3.1 is divided into the following three steps: (i) construct a series of Lyapunov functions to derive a suitable non-negative $C^{2}$-function $V(S, I, Q, R)$ and a stochastic critical value $\mathscr{R}_{0}^{S}$ related to $\mathscr{R}_{0}$; (ii) establish a reasonable bounded domain $D$ and prove the assumption $\left(\mathscr{A}_{2}\right)$ of Lemma 2.2 ; and (iii) validate the condition $\left(\mathscr{A}_{1}\right)$ of Lemma 2.2.

Step 1 Define an important $C^{2}$-function $\widetilde{V}(S, I, Q, R)$ by

$\widetilde{V}(S, I, Q, R)=M_{0}\left(-c_{0} \ln S-\ln I\right)-\ln S-\ln Q$

$$
-\ln R+\frac{1}{1+\theta}(S+I+Q+R)^{1+\theta},
$$

where $c_{0}=\frac{\Lambda \beta}{\left(\mu+\frac{\sigma_{1}^{2}}{2}\right)^{2}}>0, M>0$, and $\theta \in(0,1)$ satisfy

$$
\begin{aligned}
& \rho:=\mu-\frac{\theta}{2}\left(\sigma_{1}^{2} \vee \sigma_{2}^{2} \vee \sigma_{3}^{2} \vee \sigma_{4}^{2}\right)>0, \\
& \quad-M_{0}\left(\mu+\alpha_{1}+\gamma+\delta+\frac{\sigma_{2}^{2}}{2}\right)\left(\mathscr{R}_{0}^{S}-1\right) \\
& \quad+\bar{\lambda}=-2,
\end{aligned}
$$

with $\bar{\lambda}=\lambda+3 \mu+\alpha_{2}+\omega+\varepsilon+\frac{\sigma_{1}^{2}+\sigma_{3}^{2}+\sigma_{4}^{2}}{2}$ and $\lambda:=$ $\sup _{(S, I, Q, R) \in \mathbb{R}_{+}^{4}}\left\{\Lambda(S+I+Q+R)^{\theta}-\frac{\rho}{2}(S+I+Q+\right.$ $\left.R)^{1+\theta}\right\}$.

For simplicity, we let

$$
\begin{aligned}
& V_{1}=-c_{0} \ln S-\ln I, V_{2}=-\ln S-\ln Q-\ln R, \\
& V_{3}=\frac{1}{1+\theta}(S+I+Q+R)^{1+\theta} .
\end{aligned}
$$

By means of Itồ's formula which is shown in "Appendix C", the function $V_{1}$ satisfies

$$
\begin{aligned}
\mathscr{L} V_{1}= & c_{0}\left[-\frac{\Lambda}{S}+\beta I-\frac{\omega R}{S}\right. \\
& \left.+\left(\mu+\frac{\sigma_{1}^{2}}{2}\right)\right] \\
& +\left[-\beta S+\left(\mu+\alpha_{1}+\gamma+\delta+\frac{\sigma_{2}^{2}}{2}\right)\right] \\
\leq & \left(\mu+\alpha_{1}+\gamma+\delta+\frac{\sigma_{2}^{2}}{2}\right)+c_{0}\left(\mu+\frac{\sigma_{1}^{2}}{2}\right) \\
& +c_{0} \beta I-\left(\frac{c_{0} \Lambda}{S}+\beta S\right) \\
\leq & \left(\mu+\alpha_{1}+\gamma+\delta+\frac{\sigma_{2}^{2}}{2}\right)+c_{0}\left(\mu+\frac{\sigma_{1}^{2}}{2}\right) \\
& +c_{0} \beta I-2 \sqrt{c_{0} \Lambda \beta} \\
= & -\left(\mu+\alpha_{1}+\gamma+\delta\right. \\
& \left.+\frac{\sigma_{2}^{2}}{2}\right)\left(\mathscr{R}_{0}^{S}-1\right)+c_{0} \beta I .
\end{aligned}
$$

Employing Itô's formula to $V_{2}$, one has

$$
\begin{aligned}
\mathscr{L} V_{2}= & \left(-\frac{\Lambda}{S}+\beta I-\frac{\omega R}{S}+\mu+\frac{\sigma_{1}^{2}}{2}\right) \\
& +\left(-\frac{\delta I}{Q}+\mu+\alpha_{2}+\varepsilon+\frac{\sigma_{3}^{2}}{2}\right)+\left(-\frac{\gamma I}{R}\right. \\
& \left.-\frac{\varepsilon Q}{R}+\mu+\omega+\frac{\sigma_{4}^{2}}{2}\right) \\
\leq & -\frac{\Lambda}{S}-\frac{\delta I}{Q}-\frac{\gamma I}{R}+\beta I+3 \mu+\alpha_{2} \\
& +\omega+\varepsilon+\frac{\sigma_{1}^{2}+\sigma_{3}^{2}+\sigma_{4}^{2}}{2} .
\end{aligned}
$$

Similarly, by the definition of $\lambda$, we have

$$
\begin{aligned}
\mathscr{L} V_{3}= & (S+I+Q+R)^{\theta} \\
& {\left[\Lambda-\mu S-\left(\mu+\alpha_{1}\right) I-\left(\mu+\alpha_{2}\right) Q-\mu R\right] } \\
& +\frac{\theta}{2}(S+I+Q+R)^{\theta-1}\left(\sigma_{1}^{2} S^{2}\right. \\
& \left.+\sigma_{2}^{2} I^{2}+\sigma_{3}^{2} Q^{2}+\sigma_{4}^{2} R^{2}\right) \\
\leq & \Lambda(S+I+Q+R)^{\theta}-\mu(S+I+Q+R)^{1+\theta} \\
& +\frac{\theta}{2}\left(\sigma_{1}^{2} \vee \sigma_{2}^{2} \vee \sigma_{3}^{2} \vee \sigma_{4}^{2}\right)(S+I+Q+R)^{1+\theta} \\
\leq & \lambda-\frac{\rho}{2}(S+I+Q+R)^{1+\theta} \\
& \leq \lambda-\frac{\rho}{2}\left(S^{1+\theta}+I^{1+\theta}+Q^{1+\theta}+R^{1+\theta}\right),
\end{aligned}
$$


where $\lambda:=\sup _{(S, I, Q, R) \in \mathbb{R}_{+}^{4}}\left\{\Lambda(S+I+Q+R)^{\theta}-\right.$ $\left.\frac{\rho}{2}(S+I+Q+R)^{1+\theta}\right\}$.

In addition,

$$
\liminf _{k \rightarrow \infty,(S, I, Q, R) \in \mathbb{R}_{+}^{4} \backslash U_{k, 4}} \tilde{V}(S, I, Q, R)=+\infty .
$$

Consequently, we can construct a suitable non-negative $C^{2}$-function $V(S, I, Q, R)$ in the following form

$V(S, I, Q, R)=\widetilde{V}(S, I, Q, R)-\widetilde{V}\left(S^{0}, I^{0}, Q^{0}, R^{0}\right)$,

where $\widetilde{V}\left(S^{0}, I^{0}, Q^{0}, R^{0}\right)$ is the minimum value of $\widetilde{V}(S, I, Q, R)$.

Combining (3.1)-(3.4) and the definition of $\bar{\lambda}$, one can see that

$$
\begin{aligned}
\mathscr{L} V \leq & M_{0}\left[-\left(\mu+\alpha_{1}+\gamma+\delta+\frac{\sigma_{2}^{2}}{2}\right)\left(\mathscr{R}_{0}^{S}-1\right)\right. \\
& \left.+c_{0} \beta I\right]+\lambda+3 \mu+\alpha_{2}+\omega+\varepsilon \\
& +\frac{\sigma_{1}^{2}+\sigma_{3}^{2}+\sigma_{4}^{2}}{2}-\frac{\Lambda}{S}-\frac{\delta I}{Q} \\
& q-\frac{\gamma I}{R}+\beta I-\frac{\rho}{2}\left(S^{1+\theta}+I^{1+\theta}+Q^{1+\theta}\right. \\
& \left.+R^{1+\theta}\right)=-M_{0}\left(\mu+\alpha_{1}+\gamma+\delta\right. \\
& \left.+\frac{\sigma_{2}^{2}}{2}\right)\left(\mathscr{R}_{0}^{S}-1\right)+\bar{\lambda}+\left[\left(c_{0} M_{0}+1\right) \beta I-\frac{\rho}{2} I^{1+\theta}\right] \\
& -\frac{\Lambda}{S}-\frac{\delta I}{Q}-\frac{\gamma I}{R}-\frac{\rho}{2}\left(S^{1+\theta}+Q^{1+\theta}+R^{1+\theta}\right) \\
= & -2+\left[\left(c_{0} M_{0}+1\right) \beta I\right. \\
& \left.-\frac{\rho}{2} I^{1+\theta}\right]-\frac{\Lambda}{S}-\frac{\delta I}{Q}-\frac{\gamma I}{R} \\
& -\frac{\rho}{2}\left(S^{1+\theta}+Q^{1+\theta}+R^{1+\theta}\right) .
\end{aligned}
$$

Step 2 Consider the following bounded set

$$
\begin{aligned}
& \mathbb{D}_{\epsilon}=\left\{(S, I, Q, R) \in \mathbb{R}_{+}^{4} \mid \epsilon \leq S \leq \frac{1}{\epsilon}, \epsilon \leq I\right. \\
& \left.\leq \frac{1}{\epsilon}, \epsilon^{2} \leq Q \leq \frac{1}{\epsilon^{2}}, \epsilon^{2} \leq R \leq \frac{1}{\epsilon^{2}}\right\},
\end{aligned}
$$

where $\epsilon>0$ is a sufficiently small constant such that the following inequalities hold.

$$
\begin{aligned}
& -2+K_{1}-\frac{\rho}{2 \epsilon^{\theta+1}} \leq-1 . \\
& -2+\left(c_{0} M_{0}+1\right) \beta \epsilon \leq-1 . \\
& -2+K_{2}-\frac{\rho}{4 \epsilon^{\theta+1}} \leq-1 . \\
& -2+K_{1}-\frac{\min (\Lambda, \delta, \gamma)}{\epsilon} \leq-1 .
\end{aligned}
$$

with $K_{1}=\sup _{I \in \mathbb{R}_{+}}\left\{\left(c_{0} M_{0}+1\right) \beta I-\frac{\rho}{2} I^{1+\theta}\right\}$ and $K_{2}=\sup _{I \in \mathbb{R}_{+}}\left\{\left(c_{0} M_{0}+1\right) \beta I-\frac{\rho}{4} I^{1+\theta}\right\}$.
For simplicity, let $X(t)=(S(t), I(t), Q(t), R(t))^{\tau}$. Consider the following seven subsets of $\mathbb{R}_{+}^{4} \backslash \mathbb{D}$

$$
\begin{aligned}
& \mathbb{D}_{1, \epsilon}=\left\{X(t) \in \mathbb{R}_{+}^{4} \mid S<\epsilon\right\}, \mathbb{D}_{2, \epsilon} \\
& =\left\{X(t) \in \mathbb{R}_{+}^{4} \mid I<\epsilon\right\}, \mathbb{D}_{3, \epsilon} \\
& =\left\{X(t) \in \mathbb{R}_{+}^{4} \mid S>\frac{1}{\epsilon}\right\}, \\
& \mathbb{D}_{4, \epsilon}=\left\{X(t) \in \mathbb{R}_{+}^{4} \mid I\right. \\
& \left.>\frac{1}{\epsilon}\right\}, \mathbb{D}_{5, \epsilon}=\left\{X(t) \in \mathbb{R}_{+}^{4} \mid Q\right. \\
& \left.>\frac{1}{\epsilon} \text { or } R>\frac{1}{\epsilon}\right\} . \\
& \mathbb{D}_{6, \epsilon}=\left\{X(t) \in \mathbb{R}_{+}^{4} \mid I \geq \epsilon, Q<\epsilon^{2}\right\}, \mathbb{D}_{7, \epsilon} \\
& =\left\{X(t) \in \mathbb{R}_{+}^{4} \mid I \geq \epsilon, R<\epsilon^{2}\right\} .
\end{aligned}
$$

Clearly, $\mathbb{R}_{+}^{4} \backslash \mathbb{D}=\bigcup_{k=1}^{7} \mathbb{D}_{k, \epsilon}$. By (3.6)-(3.9), we can derive

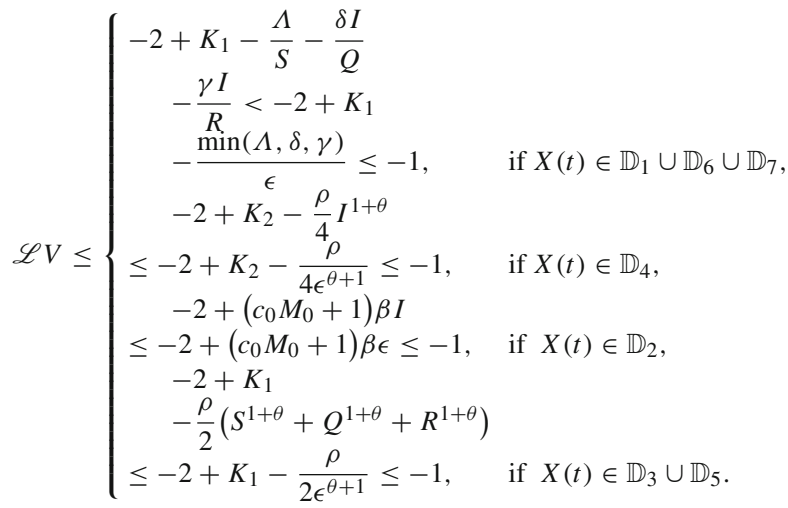

Given the above, we can therefore obtain a pair of sufficiently small $\epsilon>0$ and closed domain $\mathbb{D}_{\epsilon}$ such that $\mathscr{L} V \leq-1, \quad$ for any $(S, I, Q, R) \in \mathbb{R}_{+}^{4} \backslash \mathbb{D}_{\epsilon}$.

Hence, the assumption $\left(\mathscr{A}_{2}\right)$ of Lemma 2.2 holds.

Step 3 System (2.2) has the corresponding diffusion matrix

$$
\mathcal{F}=\left(\begin{array}{cccc}
\sigma_{1}^{2} S^{2} & 0 & 0 & 0 \\
0 & \sigma_{2}^{2} I^{2} & 0 & 0 \\
0 & 0 & \sigma_{3}^{2} Q^{2} & 0 \\
0 & 0 & 0 & \sigma_{4}^{2} R^{2}
\end{array}\right)
$$

Obviously, for any $(S, I, Q, R) \in \mathbb{D}_{\epsilon}, \mathcal{F}$ is a positive definite matrix. In other words, we can determine a positive constant $\kappa_{0}:=\inf _{X(t) \in \mathbb{D}_{\epsilon}}\left\{\sigma_{1}^{2} S^{2}, \sigma_{2}^{2} I^{2}\right.$, $\left.\sigma_{3}^{2} Q^{2}, \sigma_{4}^{2} R^{2}\right\}$ such that 


$$
\begin{aligned}
& \sum_{i=1}^{4} \sum_{j=1}^{4} \bar{a}_{i j}(S, I, Q, R) \xi_{i} \xi_{j}=\sigma_{1}^{2} S^{2} \xi_{1}^{2} \\
& \quad+\sigma_{2}^{2} I^{2} \xi_{2}^{2}+\sigma_{3}^{2} Q^{2} \xi_{3}^{2}+\sigma_{4}^{2} R^{2} \xi_{4}^{2} \geq \kappa_{0}|\xi|^{2}
\end{aligned}
$$

for any $\xi=\left(\xi_{1}, \xi_{2}, \xi_{3}, \xi_{4}\right) \in \mathbb{R}^{4}$.

Then the condition $\left(\mathscr{A}_{1}\right)$ of Lemma 2.2 also holds. Given the above three steps, system (2.2) admits a unique ergodic stationary distribution $\varpi(\cdot)$ with respect to the solution $(S(t), I(t), Q(t), R(t))$. The proof of Theorem 3.1 is confirmed.

Remark 3.2 From the expressions of $\mathscr{R}_{0}$ and $\mathscr{R}_{0}^{S}$, we can easily obtain that $\mathscr{R}_{0}^{S} \leq \mathscr{R}_{0}$. As we know, the existence of an ergodic stationary distribution denotes the stochastic positive equilibrium state. Hence, $\mathscr{R}_{0}^{S}>1$ can be regarded as the unified criterion which guarantees the disease persistence of the deterministic model (2.1) and stochastic system (2.2). Furthermore, $\mathscr{R}_{0}^{S}=$ $\mathscr{R}_{0}$ while $\sigma_{1}=\sigma_{2}=0$. This means that the disease persistence is critically affected by the random fluctuations of susceptible and infected individuals rather than quarantined and recovered individuals.

\section{Density function analyses of stationary distribution $\varpi(\cdot)$}

By Theorem 3.1, we obtain that system (2.3) has a unique stationary distribution which has ergodic property if $\mathscr{R}_{0}^{S}>1$. For further development of infectious disease dynamics, in this section, we will study the corresponding probability density function of the distribution $\varpi(\cdot)$ to derive all statistical properties of system (2.3). Before this, two equivalent differential equations of system (2.2) should be firstly introduced.

\subsection{Two important transformations of system (2.2)}

(I) (Logarithmic transformation) Let $x_{1}=\ln (S)$, $x_{2}=\ln (I), x_{3}=\ln (Q)$, and $x_{4}=\ln (R)$. By means of It $\hat{o}$ 's formula, system (2.2) can be transformed into the following equation:

$$
\left\{\begin{array}{l}
d x_{1}=\left(\Lambda e^{-x_{1}}-\mu_{1}-\beta e^{x_{2}}+\omega e^{x_{4}-x_{1}}\right) d t+\sigma_{1} d B_{1}(t) \\
d x_{2}=\left[\beta e^{x_{1}}-\left(\mu_{2}+\alpha_{1}+\delta+\gamma\right)\right]+\sigma_{2} d B_{2}(t), \\
d x_{3}=\left[\delta e^{x_{2}-x_{3}}-\left(\mu_{3}+\alpha_{2}+\varepsilon\right)\right] d t+\sigma_{3} d B_{3}(t) \\
d x_{4}=\left[\gamma e^{x_{2}-x_{4}}+\varepsilon e^{x_{3}-x_{4}}-\left(\mu_{4}+\omega\right)\right] d t+\sigma_{4} d B_{4}(t)
\end{array}\right.
$$

where $\mu_{k}=\mu+\frac{\sigma_{k}^{2}}{2}(k=1,2,3,4)$.

Assuming that $\mathscr{R}_{0}^{S}>1$ and following the description of Zhou and Zhang [33], we similarly define a quasi-endemic equilibrium $E_{+}^{*}=\left(S_{+}^{*}, I_{+}^{*}, Q_{+}^{*}, R_{+}^{*}\right)=$ $\left(e^{x_{1}^{*}}, e^{x_{2}^{*}}, e^{x_{3}^{*}}, e^{x_{4}^{*}}\right) \in \mathbb{R}_{+}^{4}$, which is determined by the following algebraic equations:

$\left\{\begin{array}{l}\Lambda e^{-x_{1}^{*}}-\mu_{1}-\beta e^{x_{2}^{*}}+\omega e^{x_{4}^{*}-x_{1}^{*}}=0, \\ \beta e^{x_{1}^{*}}-\left(\mu_{2}+\alpha_{1}+\delta+\gamma\right)=0 \\ \delta e^{x_{2}^{*}-x_{3}^{*}}-\left(\mu_{3}+\alpha_{2}+\varepsilon\right)=0, \\ \gamma e^{x_{2}^{*}-x_{4}^{*}}+\varepsilon e^{x_{3}^{*}-x_{4}^{*}}-\left(\mu_{4}+\omega\right)=0 .\end{array}\right.$

By detailed calculation, we obtain that $S_{+}^{*}=\frac{\Lambda}{\mu_{1} \mathscr{R}_{0}^{S}}$, $I_{+}^{*}=\frac{\Lambda\left(\mathscr{R}_{0}^{S}-1\right)}{\bar{\varrho}_{1} \mathscr{R}_{0}^{S}}, Q_{+}^{*}=\frac{\delta \Lambda\left(\mathscr{R}_{0}^{S}-1\right)}{\bar{\varrho}_{1}\left(\mu_{3}+\alpha_{2}+\varepsilon\right) \mathscr{R}_{0}^{S}}, R_{+}^{*}=$ $\frac{\Lambda \bar{\varrho}_{2}\left(\mathscr{R}_{0}^{S}-1\right)}{\left(\mu_{4}+\omega\right)\left(\mu_{3}+\alpha_{2}+\varepsilon\right) \mathscr{R}_{0}^{S}}$ with $\bar{\varrho}_{1}=\mu_{2}+\alpha_{1}+\frac{\mu_{4}+\gamma}{\mu_{4}+\omega}+$ $\frac{\delta\left[\left(\mu_{4}+\gamma\right)\left(\mu_{3}+\alpha_{2}\right)+\mu_{4} \varepsilon\right]}{\left(\mu_{4}+\omega\right)\left(\mu_{3}+\alpha_{2}+\varepsilon\right)}$ and $\bar{\varrho}_{2}=\gamma\left(\mu_{3}+\alpha_{2}+\varepsilon\right)+\varepsilon \delta$. In fact, the quasi-endemic equilibrium $E_{+}^{*}$ is the same as $E^{*}$ if there is no stochastic perturbation. This is the reason why the quasi-endemic equilibrium is defined. (II) (Equilibrium offset transformation) Next, by letting $Y=\left(y_{1}, y_{2}, y_{3}, y_{4}\right)^{\tau}=\left(x_{1}-x_{1}^{*}, x_{2}-x_{2}^{*}, x_{3}-\right.$ $\left.x_{3}^{*}, x_{4}-x_{4}^{*}\right)^{\tau}$, then the corresponding linearized differential equation of system (4.1) is given by

$$
\left\{\begin{array}{l}
d y_{1}=\left(-a_{11} y_{1}-a_{12} y_{2}+a_{14} y_{4}\right) d t+\sigma_{1} d B_{1}(t), \\
d y_{2}=a_{21} y_{1} d t+\sigma_{2} d B_{2}(t), \\
d y_{3}=\left(a_{32} y_{2}-a_{32} y_{3}\right) d t+\sigma_{3} d B_{3}(t), \\
d y_{4}=\left[a_{42} y_{2}+a_{43} y_{3}-\left(a_{42}+a_{43}\right) y_{4}\right] d t+\sigma_{4} d B_{4}(t),
\end{array}\right.
$$

where

$$
\begin{aligned}
& a_{11}=\frac{\Lambda+\omega R_{+}^{*}}{S_{+}^{*}}, a_{12}=\beta I_{+}^{*}, \\
& a_{14}=\frac{\omega R_{+}^{*}}{S_{+}^{*}}, a_{21}=\beta S_{+}^{*}, a_{32}=\frac{\delta I_{+}^{*}}{Q_{+}^{*}}, \\
& a_{42}=\frac{\gamma I_{+}^{*}}{R_{+}^{*}}, a_{43}=\frac{\varepsilon Q_{+}^{*}}{R_{+}^{*}} .
\end{aligned}
$$

By the definition of $E_{+}^{*}$, we easily obtain that all the parameters in (4.4) are positive constants. Next, we will study the corresponding probability density function around the quasi-endemic equilibrium $E_{+}^{*}$. 
4.2 Density function expression of stationary distribution $\varpi(\cdot)$

Theorem 4.1 Assuming that $\mathscr{R}_{0}^{S}>1$, for any initial value $(S(0), I(0), Q(0), R(0)) \in \mathbb{R}_{+}^{4}$, the solution $(S(t), I(t), Q(t), R(t))$ of the system (2.2) follows the unique log-normal probability density function $\Phi(S, I, Q, R)$ around the quasi-endemic equilibrium $E_{+}^{*}$, which is described by

$$
\begin{aligned}
& \Phi(S, I, Q, R)=(2 \pi)^{-2}|\Sigma|^{-\frac{1}{2}} \\
& e^{-\frac{1}{2}\left(\ln \frac{S}{S_{+}^{*}}, \ln \frac{I}{I_{+}^{*}}, \ln \frac{Q}{Q_{+}^{*}}, \ln \frac{R}{R_{+}^{*}}\right) \Sigma^{-1}\left(\ln \frac{S}{S_{+}^{*}}, \ln \frac{I}{I_{+}^{*}}, \ln \frac{Q}{Q_{+}^{*}}, \ln \frac{R}{R_{+}^{*}}\right)^{\tau}},
\end{aligned}
$$

where $\Sigma$ is a positive definite matrix, and the special form of $\Sigma$ is given as follows.

(1) If $w_{1} \neq 0, w_{2} \neq 0$ and $w_{3} \neq 0$, then

$$
\begin{aligned}
\Sigma= & \rho_{1}^{2}\left(M_{1} H_{1}\right)^{-1} \Sigma_{0}\left[\left(M_{1} H_{1}\right)^{-1}\right]^{\tau} \\
& +\rho_{2}^{2}\left(M_{2} H_{2} P_{2} J_{2}\right)^{-1} \Sigma_{0}\left[\left(M_{2} H_{2} P_{2} J_{2}\right)^{-1}\right]^{\tau} \\
& +\rho_{3}^{2}\left(M_{3} J_{3}\right)^{-1} \Sigma_{0}\left[\left(M_{3} J_{3}\right)^{-1}\right]^{\tau} \\
& +\rho_{4}^{2}\left(M_{4} J_{4}\right)^{-1} \Sigma_{0}\left[\left(M_{4} J_{4}\right)^{-1}\right]^{\tau} .
\end{aligned}
$$

(2) If $w_{1} \neq 0, w_{2} \neq 0$ and $w_{3}=0$, then

$$
\begin{aligned}
\Sigma= & \rho_{1}^{2}\left(M_{1} H_{1}\right)^{-1} \Sigma_{0}\left[\left(M_{1} H_{1}\right)^{-1}\right]^{\tau} \\
& +\rho_{2 w_{3}}^{2}\left(M_{2 w_{3}} H_{2} P_{2} J_{2}\right)^{-1} \widetilde{\Sigma}_{0}\left[\left(M_{2 w_{3}} H_{2} P_{2} J_{2}\right)^{-1}\right]^{\tau} \\
& +\rho_{3}^{2}\left(M_{3} J_{3}\right)^{-1} \Sigma_{0}\left[\left(M_{3} J_{3}\right)^{-1}\right]^{\tau} \\
& +\rho_{4}^{2}\left(M_{4} J_{4}\right)^{-1} \Sigma_{0}\left[\left(M_{4} J_{4}\right)^{-1}\right]^{\tau} .
\end{aligned}
$$

(3) If $w_{1} \neq 0$ and $w_{2}=0$, then

$$
\begin{aligned}
\Sigma= & \rho_{1}^{2}\left(M_{1} H_{1}\right)^{-1} \Sigma_{0}\left[\left(M_{1} H_{1}\right)^{-1}\right]^{\tau} \\
& +\rho_{2 w_{2}}^{2}\left(M_{2 w_{2}} P_{2} J_{2}\right)^{-1} \Sigma_{0}\left[\left(M_{2 w_{2}} P_{2} J_{2}\right)^{-1}\right]^{\tau} \\
& +\rho_{3}^{2}\left(M_{3} J_{3}\right)^{-1} \Sigma_{0}\left[\left(M_{3} J_{3}\right)^{-1}\right]^{\tau} \\
& +\rho_{4}^{2}\left(M_{4} J_{4}\right)^{-1} \Sigma_{0}\left[\left(M_{4} J_{4}\right)^{-1}\right]^{\tau} .
\end{aligned}
$$

(4) If $w_{1}=0$ and $w_{2} \neq 0$, then

$$
\begin{aligned}
\Sigma= & \rho_{1 w_{1}}^{2}\left(M_{w_{1}} H_{1}\right)^{-1} \widehat{\Sigma}_{0}\left[\left(M_{w_{1}} H_{1}\right)^{-1}\right]^{\tau} \\
& +\rho_{2 w_{1}}^{2}\left(M_{w_{1}} J P_{2} J_{2}\right)^{-1} \widehat{\Sigma}_{0}\left[\left(M_{w_{1}} J P_{2} J_{2}\right)^{-1}\right]^{\tau} \\
& +\rho_{3}^{2}\left(M_{3} J_{3}\right)^{-1} \Sigma_{0}\left[\left(M_{3} J_{3}\right)^{-1}\right]^{\tau} \\
& +\rho_{4}^{2}\left(M_{4} J_{4}\right)^{-1} \Sigma_{0}\left[\left(M_{4} J_{4}\right)^{-1}\right]^{\tau} .
\end{aligned}
$$

(5) If $w_{1}=0$ and $w_{2}=0$, then

$$
\begin{aligned}
\Sigma= & \rho_{1 w_{1}}^{2}\left(M_{w_{1}} H_{1}\right)^{-1} \widehat{\Sigma}_{0}\left[\left(M_{w_{1}} H_{1}\right)^{-1}\right]^{\tau} \\
& +\rho_{2 w_{12}}^{2}\left(M_{2 w_{12}} P_{2} J_{2}\right)^{-1} \bar{\Sigma}_{0}\left[\left(M_{2 w_{12}} P_{2} J_{2}\right)^{-1}\right]^{\tau} \\
& +\rho_{3}^{2}\left(M_{3} J_{3}\right)^{-1} \Sigma_{0}\left[\left(M_{3} J_{3}\right)^{-1}\right]^{\tau} \\
& +\rho_{4}^{2}\left(M_{4} J_{4}\right)^{-1} \Sigma_{0}\left[\left(M_{4} J_{4}\right)^{-1}\right]^{\tau},
\end{aligned}
$$

with

$$
\begin{aligned}
& w_{1}=\frac{\left(a_{42}+a_{43}\right)\left(a_{32}-a_{42}\right)}{a_{32}}, \\
& w_{2}=\frac{a_{11} a_{12}+a_{14} a_{42}}{a_{32}}-a_{12}, \\
& w_{3}=a_{14}+\frac{\left(a_{42}+a_{43}-a_{11}\right) w_{2}}{w_{1}}, \\
& \rho_{1}=a_{21} a_{32} w_{1} \sigma_{1}, \\
& \rho_{1 w_{1}}=a_{21} a_{32} \sigma_{1}, \rho_{2}=a_{32} w_{1} w_{3} \sigma_{2} \text {, } \\
& \rho_{2 w_{1}}=a_{32} w_{2} \sigma_{2}, \rho_{2 w_{2}}=a_{32} w_{1} \sigma_{2} \text {, } \\
& \rho_{2 w_{12}}=a_{32} \sigma_{2}, \rho_{2 w_{3}}=a_{32} w_{1} \sigma_{2} \text {, } \\
& \rho_{3}=a_{14} a_{21} a_{43} \sigma_{3}, \rho_{4}=a_{14} a_{21} a_{32} \sigma_{4} \text {, } \\
& J_{1}=\left(\begin{array}{llll}
1 & 0 & 0 & 0 \\
0 & 1 & 0 & 0 \\
0 & 0 & 0 & 1 \\
0 & 0 & 1 & 0
\end{array}\right), J_{2}=\left(\begin{array}{llll}
0 & 1 & 0 & 0 \\
0 & 0 & 1 & 0 \\
0 & 0 & 0 & 1 \\
1 & 0 & 0 & 0
\end{array}\right) \\
& J_{3}=\left(\begin{array}{llll}
0 & 0 & 1 & 0 \\
0 & 0 & 0 & 1 \\
1 & 0 & 0 & 0 \\
0 & 1 & 0 & 0
\end{array}\right), J_{4}=\left(\begin{array}{llll}
0 & 0 & 0 & 1 \\
1 & 0 & 0 & 0 \\
0 & 1 & 0 & 0 \\
0 & 0 & 1 & 0
\end{array}\right) \text {, } \\
& P_{2}=\left(\begin{array}{cccc}
1 & 0 & 0 & 0 \\
0 & 1 & 0 & 0 \\
0 & -\frac{a_{42}}{a_{32}} & 1 & 0 \\
0 & \frac{a_{12}}{a_{32}} & 0 & 1
\end{array}\right), H_{1}=\left(\begin{array}{cccc}
1 & 0 & 0 & 0 \\
0 & 1 & 0 & 0 \\
0 & 0 & 1 & 0 \\
0 & 0 & -\frac{a_{42}}{a_{32}} & 1
\end{array}\right) \\
& H_{2}=\left(\begin{array}{cccc}
1 & 0 & 0 & 0 \\
0 & 1 & 0 & 0 \\
0 & 0 & 1 & 0 \\
0 & 0 & -\frac{w_{2}}{w_{1}} & 1
\end{array}\right) \text {. }
\end{aligned}
$$

Moreover, the standardized transformation matrices $M_{1}, M_{1 w_{1}}, M_{2}, M_{2 w_{1}}, M_{2 w_{2}}, M_{2 w_{12}}, M_{2 w_{3}}, M_{3}, M_{4}$ and $\Sigma_{0}, \widehat{\Sigma}_{0}, \widetilde{\Sigma}_{0}, \bar{\Sigma}_{0}$ are described in (4.10), (4.13), (4.17), (4.24), (4.22), (4.26), (4.19), (4.30), (4.33), (4.12),(4.14), (4.21), (4.28), respectively.

Proof For the sake of convenience, by letting $G=$ $\operatorname{diag}\left(\sigma_{1}, \sigma_{2}, \sigma_{3}, \sigma_{4}\right), B(t)=\left(B_{1}(t), B_{2}(t), B_{3}(t)\right.$, $\left.B_{4}(t)\right)^{\tau}$, and 
$A=\left(\begin{array}{cccc}-a_{11} & -a_{12} & 0 & a_{14} \\ a_{21} & 0 & 0 & 0 \\ 0 & a_{32} & a_{32} & 0 \\ 0 & a_{42} & a_{43} & -\left(a_{42}+a_{43}\right)\end{array}\right)$,

then the linearized system (4.3) can be simplified to $d Y=A Y d t+G d B(t)$. According to the relevant theory of Gardiner [39], there exists a unique probability density function $\Phi\left(y_{1}, y_{2}, y_{3}, y_{4}\right)$ around the quasiendemic equilibrium $E_{+}^{*}$, which is determined by the following Fokker-Planck equation

$$
\begin{aligned}
& -\sum_{i=1}^{4} \frac{\sigma_{i}^{2}}{2} \frac{\partial^{2} \Phi}{\partial y_{i}^{2}}+\frac{\partial}{\partial y_{1}}\left[\left(-a_{11} y_{1}-a_{12} y_{2}+a_{14} y_{4}\right) \Phi\right] \\
& +\frac{\partial}{\partial y_{2}}\left(a_{21} y_{1} \Phi\right)+\frac{\partial}{\partial y_{3}}\left[\left(a_{32} y_{2}-a_{32} y_{3}\right) \Phi\right] \\
& +\frac{\partial}{\partial y_{4}}\left[\left(a_{42} y_{2}+a_{43} y_{3}-\left(a_{42}+a_{43}\right) y_{4}\right) \Phi\right]=0 .
\end{aligned}
$$

Considering the diffusion matrix $G$ is a constant matrix, from the results of Roozen [40], $\Phi\left(y_{1}, y_{2}, y_{3}, y_{4}\right)$ can be described by a Gaussian distribution. In other words,

$\Phi\left(y_{1}, y_{2}, y_{3}, y_{4}\right)=\phi_{0} e^{-\frac{1}{2}\left(y_{1}, y_{2}, y_{3}, y_{4}\right) Q\left(y_{1}, y_{2}, y_{3}, y_{4}\right)^{\tau}}$,

where $\phi_{0}$ is a positive constant, which is obtained by the normalized condition $\int_{\mathbb{R}^{4}} \Phi(Y) d y_{1} d y_{2} d y_{3} d y_{4}=1$. The real symmetric matrix $Q$ satisfies $Q G^{2} Q+A^{\tau} Q+$ $Q A=0$. If $Q$ is still a inverse matrix, let $Q^{-1}=\Sigma$. Then it is equivalent to the following equation

$G^{2}+A \Sigma+\Sigma A^{\tau}=0$.

By the finite independent superposition principle, we only need to consider the corresponding solutions of the following four algebraic sub-equations:

$G_{i}^{2}+A \Sigma_{i}+\Sigma_{i} A^{\tau}=0 \quad(i=1,2,3,4)$,

where $G_{1}=\operatorname{diag}\left(\sigma_{1}, 0,0,0\right), G_{2}=\operatorname{diag}\left(0, \sigma_{2}, 0,0\right)$, $G_{3}=\operatorname{diag}\left(0,0, \sigma_{3}, 0\right), G_{4}=\operatorname{diag}\left(0,0,0, \sigma_{4}\right)$.

Finally, we can obtain that $\Sigma=\Sigma_{1}+\Sigma_{2}+\Sigma_{3}+\Sigma_{4}$ and $G^{2}=G_{1}^{2}+G_{2}^{2}+G_{3}^{2}+G_{4}^{2}$.

Before proving the positive definiteness of $\Sigma$, we firstly verify that all the eigenvalues of $A$ have negative real parts. The corresponding characteristic polynomial of $A$ is defined by
$\varphi_{A}(\lambda)=\lambda^{4}+a_{1} \lambda^{3}+a_{2} \lambda^{2}+a_{3} \lambda+a_{4}$

where

(i) $a_{1}=a_{11}+a_{32}+a_{42}+a_{43}>0, \quad a_{2}=\left(a_{11}+\right.$ $\left.a_{32}\right)\left(a_{42}+a_{43}\right)+a_{11} a_{32}+a_{12} a_{21}>0$,

(ii) $a_{3}=\left(a_{42}+a_{43}\right)\left(a_{11} a_{32}+a_{12} a_{21}\right)+a_{12} a_{21} a_{32}-$ $a_{14} a_{21} a_{42}, \quad a_{4}=a_{21} a_{32}\left(a_{12}-a_{14}\right)\left(a_{42}+a_{43}\right)$.

From the expressions of $a_{11}, a_{12}$ and $a_{14}$, one can see that

$$
\begin{gathered}
a_{11}-a_{14}=\frac{\Lambda}{S_{+}^{*}}>0, a_{12}-a_{14}=\beta I_{+}^{*} \\
-\frac{\omega R_{+}^{*}}{S_{+}^{*}}=\frac{\beta S_{+}^{*} I_{+}^{*}-\omega R_{+}^{*}}{S_{+}^{*}} \\
=\frac{\Lambda-\mu_{1} S_{+}^{*}}{S_{+}^{*}}=\mu_{1}\left(\mathscr{R}_{0}^{S}-1\right)>0 .
\end{gathered}
$$

Therefore, it implies

(1) $a_{3}=a_{11} a_{32}\left(a_{42}+a_{43}\right)+a_{12} a_{21}\left(a_{32}+a_{43}\right)$

$$
+a_{21} a_{42}\left(a_{12}-a_{14}\right)>0,
$$

(2) $a_{4}=a_{21} a_{32}\left(a_{12}-a_{14}\right)\left(a_{42}+a_{43}\right)>0$.

Moreover, by the corresponding simplicity, we have

$$
\text { (3) } \begin{aligned}
a_{1} & \left(a_{2} a_{3}-a_{1} a_{4}\right)-a_{3}^{2} \\
= & a_{14} a_{21} a_{32}\left(a_{42}+a_{43}\right)\left(a_{11}+a_{32}+a_{42}+a_{43}\right) \\
& +a_{14} a_{21} a_{42}\left[a_{11} a_{32} a_{42}\right. \\
& \left.+2\left(a_{42}+a_{43}\right)\left(a_{11} a_{32}+a_{12} a_{21}\right)\right] \\
& +a_{11}\left(a_{11} a_{32}+a_{12} a_{21}+a_{32}^{2}\right)\left[a_{12} a_{21} a_{32}\right. \\
& +\left(a_{42}+a_{43}\right)\left(a_{11} a_{32}+a_{12} a_{21}\right)+\left(a_{42}\right. \\
& \left.\left.+a_{43}\right)^{2}\left(a_{11}+a_{32}+a_{42}+a_{43}\right)\right]-a_{14}^{2} a_{21}^{2} a_{42}^{2} \\
& -a_{14} a_{21} a_{42}\left(a_{11}+a_{32}\right)\left(a_{42}\right. \\
& \left.+a_{43}\right)\left(a_{11}+a_{32}+a_{42}+a_{43}\right) \\
& -a_{14} a_{21} a_{42}\left(a_{11} a_{32}+a_{12} a_{21}\right)\left(a_{42}\right. \\
& \left.+a_{43}\right)\left(a_{11}+a_{32}+a_{42}+a_{43}\right) \\
> & a_{11}\left(a_{11} a_{32}+a_{12} a_{21}+a_{32}^{2}\right)\left[\left(a_{42}+a_{43}\right)\right. \\
& \left.\left(a_{11} a_{32}+a_{12} a_{21}\right)+a_{21} a_{32}\left(a_{12}-a_{14}\right)\right] \\
& +a_{11}\left(a_{11}+a_{32}+a_{42}+a_{43}\right)\left(a_{42}+a_{43}\right) \\
& {\left[\left(a_{42}+a_{43}\right)\left(a_{11}+a_{32}+a_{32}^{2}\right)+a_{12} a_{21} a_{43}\right] } \\
& +a_{14} a_{21} a_{32}\left(a_{11}+a_{32}+a_{42}\right. \\
& \left.+a_{43}\right)\left(a_{42}+a_{43}\right)\left(a_{11}+a_{32}+a_{43}\right) \\
& +a_{14} a_{21} a_{42}\left[a _ { 4 3 } \left(a_{11} a_{32}\right.\right. \\
& \left.\left.+a_{12} a_{21}\right)+a_{11} a_{32} a_{42}\right]>0 \\
&
\end{aligned}
$$


which indicates that $a_{1} a_{2}-a_{3}>\frac{a_{1}^{2} a_{4}}{a_{3}}>0$. In view of the Routh-Hurwitz stability criterion [41], we can obtain that $A$ has all negative real-part eigenvalues. According to the matrix similar transformation theory, we realize that $\varphi_{A}(\lambda)$ is a similarity invariant, which means that $a_{1}, a_{2}, a_{3}$ and $a_{4}$ are also similarity invariants. where $w_{1}=\frac{\left(a_{42}+a_{43}\right)\left(a_{32}-a_{42}\right)}{a_{32}}$. Using the value of $w_{1}$, we analyze the following two cases.

Case 1 If $w_{1} \neq 0$, based on the method introduced in the subsection (I) of "Appendix B", let $B_{1}=M_{1} A_{1} M_{1}^{-1}$, where the standardized transformation matrix

$M_{1}=\left(\begin{array}{cccc}a_{21} a_{32} w_{1}-a_{32} w_{1}\left(a_{32}+a_{42}+a_{43}\right) & m_{1} & -\left(a_{42}+a_{43}\right)^{3} \\ 0 & a_{32} w_{1} & -w_{1}\left(a_{32}+a_{42}+a_{43}\right) & \left(a_{42}+a_{43}\right)^{2} \\ 0 & 0 & w_{1} & -\left(a_{42}+a_{43}\right) \\ 0 & 0 & 0 & 1\end{array}\right)$,

Next, the corresponding proof for the positive definiteness of $\Sigma$ is divided into four steps. More precisely, we will show that $\Sigma_{3}, \Sigma_{4}$ are both positive definite, and $\Sigma_{1}, \Sigma_{2}$ are both at least semi-positive definite.

Step 1 Consider the algebraic equation

$G_{1}^{2}+A \Sigma_{1}+\Sigma_{1} A^{\tau}=0$.

For the following elimination matrix $H_{1}$, by letting $A_{1}=H_{1} A H_{1}^{-1}$, we have

$$
\begin{aligned}
H_{1} & =\left(\begin{array}{llll}
1 & 0 & 0 & 0 \\
0 & 1 & 0 & 0 \\
0 & 0 & 1 & 0 \\
0 & 0 & -\frac{a_{42}}{a_{32}} & 1
\end{array}\right), \\
A_{1} & =\left(\begin{array}{cccc}
-a_{11} & -a_{12} & \frac{a_{14} a_{32}}{a_{32}} & a_{14} \\
a_{21} & 0 & 0 & 0 \\
0 & a_{32} & -a_{32} & 0 \\
0 & 0 & w_{1} & -\left(a_{42}+a_{43}\right)
\end{array}\right),
\end{aligned}
$$

with $m_{1}=w_{1}\left[\left(a_{32}+a_{42}+a_{43}\right)\left(a_{42}+a_{43}\right)+a_{32}^{2}\right]$. By direct calculation, we derive

$B_{1}=\left(\begin{array}{cccc}-a_{1} & -a_{2} & -a_{3} & -a_{4} \\ 1 & 0 & 0 & 0 \\ 0 & 1 & 0 & 0 \\ 0 & 0 & 1 & 0\end{array}\right)$.

Moreover, Eq. (4.9) can be equivalently transformed into

$$
\begin{aligned}
& \left(M_{1} H_{1}\right) G_{1}^{2}\left(M_{1} H_{1}\right)^{\tau}+B_{1}\left[\left(M_{1} H_{1}\right) \Sigma_{1}\left(M_{1} H_{1}\right)^{\tau}\right] \\
& \quad+\left[\left(M_{1} H_{1}\right) \Sigma_{1}\left(M_{1} H_{1}\right)^{\tau}\right] B_{1}^{\tau}=0 .
\end{aligned}
$$

Let $\Sigma_{0}=\rho_{1}^{-2}\left(M_{1} H_{1}\right) \Sigma_{1}\left(M_{1} H_{1}\right)^{\tau}$, where $\rho_{1}=$ $a_{21} a_{32} w_{1} \sigma_{1}$, it can be simplified as

$G_{0}^{2}+B_{1} \Sigma_{0}+\Sigma_{0} B_{1}^{\tau}=0$.

Since A has all negative real-part eigenvalues, $B_{1}$ is a standard $R_{1}$ matrix. According to Lemma 2.3, $\Sigma_{0}$ is positive definite. In the similar results described in subsection (I) of "Appendix A", the form of $\Sigma_{0}$ is given as follows.

$\Sigma_{0}=\left(\begin{array}{cccc}\frac{a_{2} a_{3}-a_{1} a_{4}}{2\left[a_{1}\left(a_{2} a_{3}-a_{1} a_{4}\right)-a_{3}^{2}\right]} & 0 & -\frac{a_{3}}{2\left[a_{1}\left(a_{2} a_{3}-a_{1} a_{4}\right)-a_{3}^{2}\right]} & 0 \\ 0 & \frac{a_{3}}{2\left[a_{1}\left(a_{2} a_{3}-a_{1} a_{4}\right)-a_{3}^{2}\right]} & 0 & -\frac{a_{1}}{2\left[a_{1}\left(a_{2} a_{3}-a_{1} a_{4}\right)-a_{3}^{2}\right]} \\ -\frac{a_{3}}{2\left[a_{1}\left(a_{2} a_{3}-a_{1} a_{4}\right)-a_{3}^{2}\right]} & 0 & \frac{a_{1}}{2\left[a_{1}\left(a_{2} a_{3}-a_{1} a_{4}\right)-a_{3}^{2}\right]} & 0 \\ 0 & -\frac{a_{1}}{2\left[a_{1}\left(a_{2} a_{3}-a_{1} a_{4}\right)-a_{3}^{2}\right]} & 0 & \frac{a_{1} a_{2}-a_{3}}{2 a_{4}\left[a_{1}\left(a_{2} a_{3}-a_{1} a_{4}\right)-a_{3}^{2}\right]}\end{array}\right)$. 
Hence, $\Sigma_{1}=\rho_{1}^{2}\left(M_{1} H_{1}\right)^{-1} \Sigma_{0}\left[\left(M_{1} H_{1}\right)^{-1}\right]^{\tau}$ is also a positive-definite matrix.

Case 2 If $w_{1}=0$, that is, $a_{32}=a_{42}$, let $B_{1 w_{1}}=$ $M_{1 w_{1}} A_{1} M_{1 w_{1}}^{-1}$, where the new standardized transformation matrix is

$M_{1 w_{1}}=\left(\begin{array}{cccc}a_{21} a_{32} & -a_{32}^{2} & a_{32}^{2} & 0 \\ 0 & a_{32} & -a_{32} & 0 \\ 0 & 0 & 1 & 0 \\ 0 & 0 & 0 & 1\end{array}\right)$

which is similarly obtained by the method in subsection (II) of "Appendix B". Then $B_{1 w_{1}}$ is

$B_{1 w_{1}}=\left(\begin{array}{cccc}-b_{1} & -b_{2} & -b_{3} & -b_{4} \\ 1 & 0 & 0 & 0 \\ 0 & 1 & 0 & 0 \\ 0 & 0 & 1 & -\left(a_{42}+a_{43}\right)\end{array}\right)$

where $b_{i}(i=1,2,3,4)$ are shorthands, and we are only concerned with their signs. Meanwhile, (4.9) can be equivalently transformed into the following equation:

$$
\begin{aligned}
& \left(M_{1 w_{1}} H_{1}\right) G_{1}^{2}\left(M_{1 w_{1}} H_{1}\right)^{\tau}+B_{1 w_{1}}\left[\left(M_{1 w_{1}} H_{1}\right) \Sigma_{1}\left(M_{1 w_{1}} H_{1}\right)^{\tau}\right] \\
& \quad+\left[\left(M_{1 w_{1}} H_{1}\right) \Sigma_{1}\left(M_{1 w_{1}} H_{1}\right)^{\tau}\right] B_{1 w_{1}}^{\tau}=0 .
\end{aligned}
$$

Let $\widehat{\Sigma}_{0}=\rho_{1 w_{1}}^{-2}\left(M_{1 w_{1}} H_{1}\right) \Sigma_{1}\left(M_{1 w_{1}} H_{1}\right)^{\tau}$, where $\rho_{1 w_{1}}=$ $a_{21} a_{32} \sigma_{1}$. We similarly obtain

$G_{0}^{2}+B_{1} \widehat{\Sigma}_{0}+\widehat{\Sigma}_{0} B_{1}^{\tau}=0$.

By means of the uniqueness of $\varphi_{A}(\lambda)$, one can see that

$$
\begin{aligned}
& \varphi_{A}(\lambda)=\varphi_{B_{1 w_{1}}}(\lambda)=\lambda^{4}+\left(b_{1}+a_{42}+a_{43}\right) \lambda^{3} \\
& \quad+\left[b_{2}+b_{1}\left(a_{42}+a_{43}\right)\right] \lambda^{2} \\
& +\left[b_{3}+b_{2}\left(a_{42}+a_{43}\right)\right] \lambda+b_{3}\left(a_{42}+a_{43}\right),
\end{aligned}
$$

which means

(i) $b_{1}=a_{1}-\left(a_{42}+a_{43}\right)=a_{11}+a_{32}>0$,

(ii) $b_{2}=a_{2}-b_{1}\left(a_{42}+a_{43}\right)$

$$
=a_{2}-\left(a_{11}+a_{32}\right)\left(a_{42}+a_{43}\right)
$$$$
=a_{11} a_{32}+a_{12} a_{21}>0 \text {, }
$$

(iii) $b_{3}=a_{3}-b_{2}\left(a_{42}+a_{43}\right)=a_{3}$

$$
\begin{gathered}
-\left(a_{11} a_{32}+a_{12} a_{21}\right)\left(a_{42}+a_{43}\right) \\
=\left(a_{12}-a_{14}\right) a_{21} a_{32}>0 .
\end{gathered}
$$

Furthermore, we can compute that

(iv) $b_{1} b_{2}-b_{3}=\left(a_{11}+a_{32}\right)\left(a_{11} a_{32}+a_{12} a_{21}\right)$

$$
\begin{aligned}
& -\left(a_{12}-a_{14}\right) a_{21} a_{32}=a_{11}\left(a_{12} a_{21}+a_{11} a_{32}\right) \\
& +a_{32}\left(a_{11} a_{32}+a_{14} a_{21}\right)>0 .
\end{aligned}
$$

Based on (i)-(iv) and Lemma 2.4, $B_{1 w_{1}}$ is a standard $R_{2}$ matrix, and $\widehat{\Sigma}_{0}$ is a semi-positive definite matrix. Considering the relevant results introduced in subsection (II) of "Appendix A", $\widehat{\Sigma}_{0}$ will be

$$
\widehat{\Sigma}_{0}=\left(\begin{array}{cccc}
\frac{b_{2}}{2\left(b_{1} b_{2}-b_{3}\right)} & 0 & -\frac{1}{2\left(b_{1} b_{2}-b_{3}\right)} & 0 \\
0 & \frac{1}{2\left(b_{1} b_{2}-b_{3}\right)} & 0 & 0 \\
-\frac{1}{2\left(b_{1} b_{2}-b_{3}\right)} & 0 & \frac{b_{1}}{2 b_{3}\left(b_{1} b_{2}-b_{3}\right)} & 0 \\
0 & 0 & 0 & 0
\end{array}\right) .
$$

Then $\Sigma_{1}=\rho_{1 w_{1}}^{2}\left(M_{1 w_{1}} H_{1}\right)^{-1} \widehat{\Sigma}_{0}\left[\left(M_{1 w_{1}} H_{1}\right)^{-1}\right]^{\tau}$ is still semi-positive definite.

Therefore, given the above cases, we get that $\Sigma_{1}$ is at least semi-positive definite.

Step 2 For the following algebraic equation

$G_{2}^{2}+A \Sigma_{2}+\Sigma_{2} A^{\tau}=0$,

consider the corresponding order matrix $J_{2}$ and elimination matrix $P_{2}$ :

$J_{2}=\left(\begin{array}{llll}0 & 1 & 0 & 0 \\ 0 & 0 & 1 & 0 \\ 0 & 0 & 0 & 1 \\ 1 & 0 & 0 & 0\end{array}\right), \quad P_{2}=\left(\begin{array}{cccc}1 & 0 & 0 & 0 \\ 0 & 1 & 0 & 0 \\ 0 & -\frac{a_{42}}{a_{32}} & 1 & 0 \\ 0 & \frac{a_{12}}{a_{32}} & 0 & 1\end{array}\right)$.

Let $B_{2}=\left(P_{2} J_{2}\right) A\left(P_{2} J_{2}\right)^{-1}$. Then we can obtain that

$$
B_{2}=\left(\begin{array}{cccc}
0 & -\frac{a_{12} a_{21}}{a_{32}} & 0 & a_{21} \\
a_{32} & -a_{32} & 0 & 0 \\
0 & w_{1} & -\left(a_{42}+a_{43}\right) & 0 \\
0 & w_{2} & a_{14} & -a_{11}
\end{array}\right),
$$

where $w_{1}, w_{2}$ are the same as those in Theorem 4.1. Similarly, based on the values of $w_{1}$ and $w_{2}$, the following four sub-cases shall be analyzed.

$$
\begin{aligned}
& \left(\mathbf{I}_{1}\right) w_{1} \neq 0, w_{2} \neq 0 ; \quad\left(\mathbf{I}_{2}\right) \\
& \quad w_{1} \neq 0, w_{2}=0 ; \quad\left(\mathbf{I}_{3}\right) \\
& w_{1}=0, w_{2} \neq 0 ; \quad\left(\mathbf{I}_{4}\right) w_{1}=0, w_{2}=0 .
\end{aligned}
$$

Case $\left(I_{1}\right)$ For the following elimination matrix $\mathrm{H}_{2}$, let $C_{2}=\mathrm{H}_{2} \mathrm{~B}_{2} \mathrm{H}_{2}^{-1}$ :

$$
H_{2}=\left(\begin{array}{cccc}
1 & 0 & 0 & 0 \\
0 & 1 & 0 & 0 \\
0 & 0 & 1 & 0 \\
0 & 0 & -\frac{w_{2}}{w_{1}} & 1
\end{array}\right)
$$




$$
C_{2}=\left(\begin{array}{cccc}
0 & -\frac{a_{12} a_{21}}{a_{32}} & \frac{a_{21} w_{2}}{w_{1}} & a_{21} \\
a_{32} & -a_{32} & 0 & 0 \\
0 & w_{1} & -\left(a_{42}+a_{43}\right) & 0 \\
0 & 0 & w_{3} & -a_{11}
\end{array}\right)
$$

where $w_{3}=a_{14}+\frac{\left(a_{42}+a_{43}-a_{11}\right) w_{2}}{w_{1}}$. Based on the value of $w_{3}$, we will discuss the following two sub-cases of

Case $\left(I_{1}\right)$

- If $w_{3} \neq 0$, in the similar method as Case $\mathbf{1}$ of Step 1 described, we construct the following standardized transformation matrix:
Similarly, $\widetilde{b}_{i}(i=1,2,3,4)$ are shorthands, and we only focus on their signs. According to the method presented in Case 2 of Step 1, the characteristic polynomial $A$ is described by

$$
\begin{aligned}
& \varphi_{A}(\lambda)=\varphi_{D_{2 w_{3}}}(\lambda) \\
& =\lambda^{4}+\left(\widetilde{b}_{1}+a_{11}\right) \lambda^{3} \\
& \quad+\left(\widetilde{b}_{2}+\widetilde{b}_{1} a_{11}\right) \lambda^{2}+\left(\widetilde{b}_{3}+\widetilde{b}_{2} a_{11}\right) \lambda+\widetilde{b}_{3} a_{11},
\end{aligned}
$$

where

(i) $\widetilde{b}_{1}=a_{1}-a_{11}=a_{32}+a_{42}+a_{43}>0$,

$M_{2}=\left(\begin{array}{cccc}a_{32} w_{1} w_{3}-w_{1} w_{3}\left(a_{11}+a_{32}+a_{42}+a_{43}\right) & m_{2} & -a_{11}^{3} \\ 0 & w_{1} w_{3} & -w_{3}\left(a_{11}+a_{42}+a_{43}\right) & a_{11}^{2} \\ 0 & 0 & w_{3} & -a_{11} \\ 0 & 0 & 0 & 1\end{array}\right)$,

where $m_{2}=w_{3}\left[\left(a_{11}+a_{42}+a_{43}\right)\left(a_{42}+a_{43}\right)+a_{11}^{2}\right]$. Considering the similar invariant properties of $a_{1}, a_{2}$, $a_{3}, a_{4}$, we can clearly derive that the standard $R_{1}$ matrix of $A$ is unique. From the results shown in subsection (I) of "Appendix B", let $D_{2}=M_{2} C_{2} M_{2}^{-1}$. Then we obtain that $D_{2}=B_{1}$, and (4.15) can be transformed into

$G_{0}^{2}+D_{2} \Sigma_{0}+\Sigma_{0} D_{2}^{\tau}=0$

where $\Sigma_{0}=\rho_{2}^{-2}\left(M_{2} H_{2} P_{2} J_{2}\right) \Sigma_{2}\left(M_{2} H_{2} P_{2} J_{2}\right)^{-1}$ with $\rho_{2}=a_{32} w_{1} w_{3} \sigma_{2}$.

Consequently, $\Sigma_{2}=\rho_{2}^{2}\left(M_{2} H_{2} P_{2} J_{2}\right)^{-1} \Sigma_{0}\left[\left(M_{2} H_{2} P_{2}\right.\right.$ $\left.\left.J_{2}\right)^{-1}\right]^{\tau}$ is a positive definite matrix.

- If $w_{3}=0$, the relevant standardized transformation matrix is given as follows:

$M_{2 w_{3}}=\left(\begin{array}{cccc}a_{32} w_{1}-w_{1}\left(a_{32}+a_{42}+a_{43}\right) & \left(a_{42}+a_{43}\right)^{2} & 0 \\ 0 & w_{1} & -\left(a_{42}+a_{43}\right) & 0 \\ 0 & 0 & 1 & 0 \\ 0 & 0 & 0 & 1\end{array}\right)$.

Define $D_{2 w_{3}}=M_{2 w_{3}} C_{2} M_{2 w_{3}}^{-1}$. By simple computation, we have

$D_{2 w_{3}}=\left(\begin{array}{cccc}-\widetilde{b}_{1} & -\widetilde{b}_{2} & -\widetilde{b}_{3} & -\widetilde{b}_{4} \\ 1 & 0 & 0 & 0 \\ 0 & 1 & 0 & 0 \\ 0 & 0 & 1 & -a_{11}\end{array}\right)$ (ii) $\widetilde{b}_{2}=a_{2}-\widetilde{b}_{1} a_{11}$

$$
\begin{aligned}
& =a_{2}-a_{11}\left(a_{32}+a_{42}+a_{43}\right) \\
& =a_{32}\left(a_{42}+a_{43}\right)+a_{12} a_{21}>0,
\end{aligned}
$$

(iii) $\widetilde{b}_{3}=\frac{a_{4}}{a_{11}}$

$$
=\frac{a_{21} a_{32}\left(a_{12}-a_{14}\right)\left(a_{42}+a_{43}\right)}{a_{11}}>0 .
$$

Moreover, noting that

$$
\begin{gathered}
\widetilde{b}_{3}=a_{3}-\widetilde{b}_{2} a_{11}=a_{3}-a_{11}\left[a_{32}\left(a_{42}+a_{43}\right)+a_{12} a_{21}\right] \\
=a_{12} a_{21}\left(a_{32}+a_{42}+a_{43}\right)-a_{21}\left(a_{11} a_{12}+a_{14} a_{32}\right),
\end{gathered}
$$

we can obtain

$$
\begin{aligned}
& \text { (iv) } \widetilde{b}_{1} \widetilde{b}_{2}-\widetilde{b}_{3} \\
& =\left(a_{32}+a_{42}+a_{43}\right)\left[a_{32}\left(a_{42}+a_{43}\right)\right. \\
& \left.+a_{12} a_{21}\right]-a_{12} a_{21}\left(a_{32}+a_{42}+a_{43}\right) \\
& +a_{21}\left(a_{11} a_{12}+a_{14} a_{32}\right) \\
& =a_{32}\left(a_{42}+a_{43}\right)\left(a_{32}+a_{42}\right. \\
& \left.+a_{43}\right)+a_{21}\left(a_{11} a_{12}+a_{14} a_{32}\right)>0 \text {. }
\end{aligned}
$$

Hence, $D_{2 w_{3}}$ is a standard $R_{2}$ matrix. Meanwhile, we can transform (4.15) into the following equation:

$G_{0}^{2}+D_{2 w_{3}} \widetilde{\Sigma}_{0}+\widetilde{\Sigma}_{0} D_{2 w_{3}}^{\tau}=0$,

where $\widetilde{\Sigma}_{0}=\rho_{2 w_{3}}^{-2}\left(M_{2 w_{3}} H_{2} P_{2} J_{2}\right) \Sigma_{2}\left(M_{2 w_{3}} H_{2} P_{2} J_{2}\right)^{-1}$ with $\rho_{2 w_{1}}=a_{32} w_{1} \sigma_{2}$. By means of Lemma 2.4 and the 
above results (i)-(iv), it is found that $\widetilde{\Sigma}_{0}$ is semi-positive definite. Following the detailed results described in subsection (II) of "Appendix A", we derive

$$
\widetilde{\Sigma}_{0}=\left(\begin{array}{cccc}
\frac{\widetilde{b}_{2}}{2\left(\widetilde{b}_{1} \vec{b}_{2}-\widetilde{b}_{3}\right)} & 0 & -\frac{1}{2\left(\widetilde{b}_{1} \widetilde{b}_{2}-\widetilde{b}_{3}\right)} & 0 \\
0 & \frac{1}{2\left(b_{1} b_{2}-\breve{b}_{3}\right)} & 0 & 0 \\
-\frac{\widetilde{b}_{1}}{2\left(\widetilde{b}_{1} \vec{b}_{2}-\widetilde{b}_{3}\right)} & 0 & \frac{\widetilde{b}_{1}}{2 \widetilde{b}_{3}\left(b_{1} b_{2}-\widetilde{b}_{3}\right)} & 0 \\
0 & 0 & 0 & 0
\end{array}\right)
$$

Thus, $\quad \Sigma_{2}=\rho_{2 w_{3}}^{2}\left(M_{2 w_{3}} H_{2} P_{2} J_{2}\right)^{-1} \widetilde{\Sigma}_{0}\left[\left(M_{2 w_{3}}\right.\right.$ $\left.\left.\mathrm{H}_{2} \mathrm{P}_{2} \mathrm{~J}_{2}\right)^{-1}\right]^{\tau}$ is semi-positive definite.

Case $\left(I_{2}\right)$ If $w_{1} \neq 0$ and $w_{2}=0$, consider the following standardized transformation matrix:

$M_{2 w_{2}}=\left(\begin{array}{cccc}a_{14} a_{32} w_{1} & -a_{14} w_{1}\left(a_{11}+a_{32}+a_{42}+a_{43}\right) & m_{3} & -a_{11}^{3} \\ 0 & a_{14} w_{1} & -a_{14}\left(a_{11}+a_{42}+a_{43}\right) & a_{11}^{2} \\ 0 & 0 & a_{14} & -a_{11} \\ 0 & 0 & 0 & 1\end{array}\right)$,
We can then define $D_{2 w_{1}}=M_{2 w_{1}} C_{2 w_{1}} M_{2 w_{1}}^{-1}$, where the standardized transformation matrix $M_{2 w_{1}}$ is given by

$$
M_{2 w_{1}}=\left(\begin{array}{cccc}
a_{32} w_{2}-w_{2}\left(a_{11}+a_{32}\right) & a_{11}^{2} & -a_{14}\left(a_{11}+a_{42}+a_{43}\right) \\
0 & w_{2} & -a_{11} & a_{14} \\
0 & 0 & 1 & 0 \\
0 & 0 & 0 & 1
\end{array}\right) .
$$

Based on subsection (II) of "Appendix B", $D_{2 w_{1}}$ is also a standard $R_{2}$ matrix, which satisfies

$D_{2 w_{1}}=\left(\begin{array}{cccc}-d_{1} & -d_{2} & -d_{3} & -d_{4} \\ 1 & 0 & 0 & 0 \\ 0 & 1 & 0 & 0 \\ 0 & 0 & 1 & -\left(a_{42}+a_{43}\right)\end{array}\right)$ where $m_{3}=a_{14}\left[\left(a_{11}+a_{42}+a_{43}\right)\left(a_{42}+a_{43}\right)+a_{11}^{2}\right]$.

Let $D_{2 w_{2}}=M_{2 w_{2}} B_{2} M_{2 w_{2}}^{-1}$. From the descriptions in subsection (I) of "Appendix B", then $D_{2 w_{2}}$ is also a standard $R_{1}$ matrix. Based on the uniqueness of the standard $R_{1}$ matrix of $A, D_{2 w_{2}}=B_{1}$. Thus, (4.15) can be converted to the equivalent equation

$G_{0}^{2}+D_{2 w_{2}} \Sigma_{0}+\Sigma_{0} D_{2 w_{2}}^{\tau}=0$

where $\Sigma_{0}=\rho_{2 w_{2}}^{-2}\left(M_{2 w_{2}} H_{2} P_{2} J_{2}\right) \Sigma_{2}\left(M_{2 w_{2}} H_{2} P_{2} J_{2}\right)^{-1}$ with $\rho_{2 w_{2}}=a_{32} w_{1} \sigma_{2}$.

Consequently, $\Sigma_{2}$ is a positive-definite matrix, and $\Sigma_{2}=\rho_{2 w_{2}}^{2}\left(M_{2 w_{2}} H_{2} P_{2} J_{2}\right)^{-1} \Sigma_{0}\left[\left(M_{2 w_{2}} H_{2} P_{2} J_{2}\right)^{-1}\right]^{\tau}$.

Case $\left(I_{3}\right)$ If $w_{1}=0$ and $w_{2} \neq 0$, for the following order matrix $J_{1}$, by letting $C_{2 w_{1}}=J_{1} B_{2} J_{1}^{-1}$, we find that

$$
\begin{aligned}
J_{1} & =\left(\begin{array}{llll}
1 & 0 & 0 & 0 \\
0 & 1 & 0 & 0 \\
0 & 0 & 0 & 1 \\
0 & 0 & 1 & 0
\end{array}\right), \\
C_{2 w_{1}} & =\left(\begin{array}{cccc}
0 & -\frac{a_{12} a_{21}}{a_{32}} & a_{21} & 0 \\
a_{32} & -a_{32} & 0 & 0 \\
0 & w_{2} & -a_{11} & a_{14} \\
0 & 0 & 0 & -\left(a_{42}+a_{43}\right)
\end{array}\right) .
\end{aligned}
$$

where $d_{j}(j=1,2,3,4)$ are shorthands. From the result of

$$
\begin{aligned}
& \varphi_{A}(\lambda)=\varphi_{D_{2 w_{1}}}(\lambda) \\
& =\left[\lambda-\left(a_{42}+a_{43}\right)\right]\left(\lambda^{3}+d_{1} \lambda^{2}+d_{2} \lambda+d_{3}\right),
\end{aligned}
$$

it indicates that $\left(d_{1}, d_{2}, d_{3}\right)^{\tau}=\left(b_{1}, b_{2}, b_{3}\right)^{\tau}$, which is the same as in Case 2 of Step 1. Thus, (4.15) can be transformed into the following equation:

$G_{0}^{2}+D_{2 w_{1}} \widehat{\Sigma}_{0}+\widehat{\Sigma}_{0} D_{2 w_{1}}^{\tau}=0$,

where $\widehat{\Sigma}_{0}=\rho_{2 w_{1}}^{-2}\left(M_{2 w_{1}} J_{1} H_{2} P_{2} J_{2}\right) \Sigma_{2}\left(M_{2 w_{2}} J_{1} H_{2}\right.$ $\left.P_{2} J_{2}\right)^{-1}$ with $\rho_{2 w_{2}}=a_{32} w_{2} \sigma_{2}$.

By the property of $\widehat{\Sigma}_{0}$, then $\Sigma_{2}=\left(M_{2 w_{2}} J_{1} H_{2} P_{2} J_{2}\right)^{-1}$ $\widehat{\Sigma}_{0}\left[\left(M_{2 w_{2}} J_{1} H_{2} P_{2} J_{2}\right)^{-1}\right]^{\tau}$ is semi-positive definite matrix.

Case (I $\left.I_{4}\right)$ If $w_{1}=0$ and $w_{2}=0$, i.e., $a_{11} a_{22}=\left(a_{12}-\right.$ $\left.a_{14}\right) a_{32}$, then using a similar method as in subsection (III) of "Appendix B", let $D_{2 w_{12}}=M_{2 w_{12}} B_{2} M_{2 w_{12}}^{-1}$, where the corresponding standardized transformation matrix $M_{2 w_{12}}$ is given by

$M_{2 w_{12}}=\left(\begin{array}{cccc}-a_{32}^{2} & a_{32}^{2}-a_{12} a_{21} & 0 & a_{21} a_{32} \\ a_{32} & -a_{32} & 0 & 0 \\ 0 & 0 & 1 & 0 \\ 0 & 0 & 0 & 1\end{array}\right)$ 
Then we have

$D_{2 w_{12}}=\left(\begin{array}{cccc}-c_{1}-c_{2} & -c_{3} & -c_{4} \\ 1 & 0 & 0 & 0 \\ 0 & 0 & -\left(a_{42}+a_{43}\right) & 0 \\ 0 & 0 & a_{14} & -a_{11}\end{array}\right)$,

where $c_{k}(k=1,2,3,4)$ are shorthands. On account of the similarity invariant of $\varphi_{A}(\lambda)$, one has

$$
\begin{aligned}
& \varphi_{A}(\lambda)=\varphi_{D_{2 w_{12}}}(\lambda) \\
& =\lambda^{4}+\left(c_{1}+a_{11}+a_{42}+a_{43}\right) \lambda^{3} \\
& \quad+\left[c_{2}-c_{1}\left(a_{11}+a_{42}+a_{43}\right)+a_{11}\left(a_{42}+a_{43}\right)\right] \lambda^{2}
\end{aligned}
$$

Consequently, $\quad \Sigma_{2}=\rho_{2 w_{3}}^{2}\left(M_{2 w_{3}} H_{2} P_{2} J_{2}\right)^{-1}$ $\bar{\Sigma}_{0}\left[\left(M_{2 w_{3}} H_{2} P_{2} J_{2}\right)^{-1}\right]^{\tau}$ is also semi-positive definite matrix.

Given the above analyses, $\Sigma_{2}$ is at least a semi-positive definite matrix.

Step 3 Consider the following algebraic equation:

$G_{3}^{2}+A \Sigma_{3}+\Sigma_{3} A^{\tau}=0$.

Let $B_{3}=\left(M_{3} J_{3}\right) A\left(M_{3} J_{3}\right)^{-1}$, where the relevant order matrix $J_{3}$ is the same as that described in Theorem 4.1, and standardized transformation matrix $M_{3}$ is given by

$$
M_{3}=\left(\begin{array}{cccc}
a_{14} a_{21} a_{43} & -a_{14} a_{21}\left(a_{11}+a_{42}+a_{43}\right) & a_{21}\left(a_{11}^{2}-a_{12} a_{21}\right) & a_{21}\left(a_{11} a_{12}+a_{14} a_{42}\right) \\
0 & a_{14} a_{21} & -a_{11} a_{21} & -a_{12} a_{21} \\
0 & 0 & a_{21} & 0 \\
0 & 0 & 0 & 1
\end{array}\right) .
$$

$$
\begin{aligned}
& +\left[c_{1} a_{11}\left(a_{42}+a_{43}\right)-c_{2}\left(a_{11}+a_{42}+a_{43}\right)\right] \lambda \\
& +c_{2} a_{11}\left(a_{42}+a_{43}\right),
\end{aligned}
$$

which implies

(i) $c_{1}=a_{1}-\left(a_{11}+a_{42}+a_{43}\right)=a_{32}>0$,

(ii) $c_{2}=\frac{a_{4}}{a_{11}\left(a_{42}+a_{43}\right)}$

$$
\begin{aligned}
& =\frac{a_{21} a_{32}\left(a_{12}-a_{14}\right)\left(a_{42}+a_{43}\right)}{a_{11}\left(a_{42}+a_{43}\right)} \\
& =\frac{a_{21} a_{32}\left(a_{12}-a_{14}\right)}{a_{11}}=a_{12} a_{21}>0 .
\end{aligned}
$$

With Lemma 2.5, we obtain that $D_{2 w_{12}}$ is a standard $R_{3}$ matrix. Additionally, by letting $\bar{\Sigma}_{0}=\rho_{2 w_{12}}^{-2}\left(M_{2 w_{12}}\right.$ $\left.P_{2} J_{2}\right) \Sigma_{2}\left(M_{2 w_{12}} P_{2} J_{2}\right)^{-1}$ with $\rho_{2 w_{12}}=a_{32} \sigma_{2}$, then (4.15) can be equivalently transformed into the following equation:

$G_{0}^{2}+D_{2 w_{12}} \bar{\Sigma}_{0}+\bar{\Sigma}_{0} D_{2 w_{12}}^{\tau}=0$.

Following Lemma 2.5 and the results described in subsection (III) of "Appendix A", $\bar{\Sigma}_{0}$ is a semi-positive definite matrix, which has the following form:

$\bar{\Sigma}_{0}=\left(\begin{array}{cccc}\frac{1}{2 c_{1}} & 0 & 0 & 0 \\ 0 & \frac{1}{2 c_{1} c_{2}} & 0 & 0 \\ 0 & 0 & 0 & 0 \\ 0 & 0 & 0 & 0\end{array}\right)$
Similarly, we get that $B_{3}$ is a standard $R_{1}$ matrix. Given the uniqueness, then $B_{3}=B_{1}$. Furthermore, Eq. (4.29) equivalently converts to the following equation:

$G_{0}^{2}+B_{3} \Sigma_{0}+\Sigma_{0} B_{3}^{\tau}=0$,

where $\Sigma_{0}=\rho_{3}^{-2}\left(M_{3} J_{3}\right) \Sigma_{3}\left(M_{3} J_{3}\right)^{-1}$ with $\rho_{3}=$ $a_{14} a_{21} a_{43} \sigma_{3}$.

By the property of $\Sigma_{0}$, then $\Sigma_{3}=\left(M_{3} J_{3}\right)^{-1}$ $\Sigma_{0}\left[\left(M_{3} J_{3}\right)^{-1}\right]^{\tau}$ is positive definite.

Step 4 Consider the following algebraic equation:

$G_{4}^{2}+A \Sigma_{4}+\Sigma_{4} A^{\tau}=0$.

Define $B_{4}=\left(M_{4} J_{4}\right) A\left(M_{4} J_{4}\right)^{-1}$, where the corresponding order matrix $J_{4}$ is shown in Theorem 4.1, and standardized transformation matrix $M_{4}$ is described by

$$
M_{4}=\left(\begin{array}{cccc}
a_{14} a_{21} a_{32} & -a_{21} a_{32}\left(a_{11}+a_{32}\right) & a_{32}\left(a_{32}^{2}-a_{12} a_{21}\right) & -a_{32}^{3} \\
0 & a_{21} a_{32} & -a_{32}^{2} & a_{32}^{2} \\
0 & 0 & a_{32} & -a_{32} \\
0 & 0 & 0 & 1
\end{array}\right) .
$$

Obviously, $B_{4}$ is also a standard $R_{1}$ matrix, which means that $B_{4}=B_{1}$. Similarly, (4.32) is equivalent to the following algebraic equation: 
$G_{0}^{2}+B_{4} \Sigma_{0}+\Sigma_{0} B_{4}^{\tau}=0$,

where $\Sigma_{0}=\rho_{4}^{-2}\left(M_{4} J_{4}\right) \Sigma_{4}\left(M_{4} J_{4}\right)^{-1}$ with $\rho_{4}=$ $a_{14} a_{21} a_{32} \sigma_{4}$.

Consequently, $\Sigma_{4}$ is positive definite, and $\Sigma_{4}=$ $\left(M_{4} J_{4}\right)^{-1} \Sigma_{0}\left[\left(M_{4} J_{4}\right)^{-1}\right]^{\tau}$.

In summary, $\Sigma_{3}, \Sigma_{4}$ are both positive definite. Furthermore, $\Sigma_{1}, \Sigma_{2}$ are both at least semi-positive definite. Hence, $\Sigma=\Sigma_{1}+\Sigma_{2}+\Sigma_{3}+\Sigma_{4}$ is a positive definite matrix, and the corresponding special form of $\Sigma$ can be determined by the above steps. By the positive definiteness of $\Sigma$, we can compute that $\phi_{0}=(2 \pi)^{-2}|\Sigma|^{-\frac{1}{2}}$. Thus, there exists a unique exact normal density function around the quasi-endemic equilibrium $E_{+}^{*}$ while $\mathscr{R}_{0}^{S}>1$, which is given by $\Phi\left(y_{1}, y_{2}, y_{3}, y_{4}\right)=(2 \pi)^{-2}|\Sigma|^{-\frac{1}{2}} \exp \left(-\frac{1}{2}\left(y_{1}\right.\right.$, $\left.\left.y_{2}, y_{3}, y_{4}\right) \Sigma^{-1}\left(y_{1}, y_{2}, y_{3}, y_{4}\right)^{\tau}\right)$. Considering the transformation of $\left(y_{1}, y_{2}, y_{3}, y_{4}\right)$ and $(S, I, Q, R)$, then the unique ergodic stationary distribution $\varpi(\cdot)$ of system (2.2) approximately follows a unique log-normal probability density function

$$
\begin{aligned}
& \Phi(S, I, Q, R)=(2 \pi)^{-2}|\Sigma|^{-\frac{1}{2}} \\
& e^{-\frac{1}{2}\left(\ln \frac{S}{S_{+}^{*}}, \ln \frac{I}{I_{+}^{*}}, \ln \frac{Q}{Q_{+}^{*}}, \ln \frac{R}{R_{+}^{*}}\right) \Sigma^{-1}\left(\ln \frac{S}{S_{+}^{*}}, \ln \frac{I}{I_{+}^{*}}, \ln \frac{Q}{Q_{+}^{*}}, \ln \frac{R}{R_{+}^{*}}\right)^{\tau} .} .
\end{aligned}
$$

This completes the proof of Theorem 4.1.

Remark 4.2 By means of Theorems 3.1 and 4.1, if $\mathscr{R}_{0}^{S}>1$, we can obtain that the unique ergodic stationary distribution $\varpi(\cdot)$ of system (2.2) admits the corresponding probability density function $\Phi(S, I, Q, R)$. Hence, $\mathscr{R}_{0}^{S}>1$ can be considered as a reasonable stochastic criterion for the disease persistence, and the exact expression of the density function of system (2.2) can provide an effective method to prevent and control many epidemics.

\section{Numerical simulations and parameter analyses}

In this section, we will introduce some examples and simulations to illustrate the above theoretical results. Making use of the common higher-order method developed by Milstein [41], the corresponding discretization equation of system (2.2) is given by

$$
\left\{\begin{array}{c}
S^{k+1}=S^{k}+\left(\Lambda-\mu S^{k}-\beta S^{k} I^{k}+\omega R^{k}\right) \Delta t \\
+\sigma_{1} S^{k} \sqrt{\Delta t} \xi_{k}+\frac{\sigma_{1}^{2}}{2} S^{k}\left(\xi_{k}^{2}-1\right) \Delta t, \\
I^{k+1}=I^{k}+\left[\beta S^{k} I^{k}-\left(\mu+\alpha_{1}+\gamma+\delta\right) I^{k}\right] \Delta t \\
\quad+\sigma_{2} I^{k} \sqrt{\Delta t} \eta_{k}+\frac{\sigma_{2}^{2}}{2} I^{k}\left(\eta_{k}^{2}-1\right) \Delta t, \\
Q^{k+1}=Q^{k}+\left[\delta I^{k}-\left(\mu+\alpha_{2}+\varepsilon\right) Q^{k}\right] \Delta t \\
+\sigma_{3} Q^{k} \sqrt{\Delta t} \zeta_{k}+\frac{\sigma_{3}^{2}}{2} Q^{k}\left(\zeta_{k}^{2}-1\right) \Delta t, \\
R^{k+1}=R^{k}+\left[\gamma I^{k}+\varepsilon Q^{k}-(\mu+\omega) R^{k}\right] \Delta t \\
+\sigma_{4} R^{k} \sqrt{\Delta t} v_{k}+\frac{\sigma_{4}^{2}}{2} R^{k}\left(v_{k}^{2}-1\right) \Delta t,
\end{array}\right.
$$

where the time increment is $\Delta t>0$, and $\xi_{k}, \eta_{k}$, $\zeta_{k}, v_{k}$ are the independent Gaussian random variables which follow the Gaussian distribution $N(0,1)$ for $k=1,2, \ldots, n$. From the realistic statistics described by Hethcote [15], Qi [34] and the Central Statistical Office of Zimbabwe (CSZ), the corresponding biological parameters and initial value of system (2.2) are shown in Table 1.

Next, based on Table 1, we will perform some empirical examples to focus on the following four aspects:

(i) The ergodicity property and the existence of a unique stationary distribution if $\mathscr{R}_{0}^{S}>1$.

(ii) The corresponding dynamical behavior of system (2.2) under $\mathscr{R}_{0}^{S} \leq 1$.

(iii) The influence of stochastic fluctuations on the disease persistence of system (2.2).

(iv) The effects of the main parameters of system (2.2) on the disease dynamics, such as the recruitment rate and transmission rate.

In addition, we still give the exact expression of a unique log-normal density function $\Phi(S, I, Q, R)$ with respect to the distribution $\varpi(\cdot)$.

5.1 Dynamical behaviors of system (2.2) under $\mathscr{R}_{0}^{S}>1$

Example 5.1 According to Table 1, let the main parameters $\left(\Lambda, \beta, \mu, \alpha_{1}, \alpha_{2}, \delta, \gamma, \varepsilon, \omega\right)=(25000$, $\left.4 \times 10^{-6}, 0.0143,0.02,0.01,0.2,0.2,0.15,0.25\right)$ and let the stochastic perturbations $\left(\sigma_{1}, \sigma_{2}, \sigma_{3}, \sigma_{4}\right)=$ $(0.008,0.004,0.005,0.005)$. We then calculate that

$$
\begin{aligned}
& \mathscr{R}_{0}=\frac{\Lambda \beta}{\mu\left(\mu+\alpha_{1}+\delta+\gamma\right)}=16.1184>1, \\
& \mathscr{R}_{0}^{S}=\frac{\Lambda \beta}{\left(\mu+\frac{\sigma_{1}^{2}}{2}\right)\left(\mu+\alpha_{1}+\gamma+\delta+\frac{\sigma_{2}^{2}}{2}\right)}=16.0821>1 .
\end{aligned}
$$


Table 1 List of biological parameters and initial value of system (2.2)

\begin{tabular}{lllll}
\hline Parameters & Description & Unit & Value & Source \\
\hline$\Lambda$ & Recruitment rate of the population & Per year & $\geq 10,000$ people & {$[34]$} \\
$\beta$ & Transmission rate of the susceptible individuals & Per year & {$[0,1] \times 10^{-5}$} & {$[34]$} \\
$\mu$ & Natural death rate of the population & Per year & {$[0.01,0.025]$} & {$[15]$, CSZ data } \\
$\alpha_{1}$ & Disease mortality of the infected individuals & Per year & {$[0,0.1]$} & {$[15,34]$} \\
$\alpha_{2}$ & Disease mortality of the quarantined individuals & Per year & {$[0,0.08]$} & {$[15]$} \\
$\delta$ & Quarantine coefficient & Per year & {$[0.1,4]$} & {$[34]$} \\
$\gamma$ & Recovered rate of the infected people & Per year & {$[0.1,2]$} & {$[34]$} \\
$\varepsilon$ & Recovered rate of the quarantined individuals & Per year & {$[0.1,0.4]$} & {$[15]$} \\
$\omega$ & Immune loss rate of the recovered individuals & Per year & {$[0.1,0.3]$} & Estimated \\
$Z(0)$ & The initial value of system (2.2) & Million & $(0.1,0.28,0.32,0.4)$ & Estimated \\
\hline
\end{tabular}

By Theorem 3.1, we can obtain that system (2.3) has a unique stationary distribution $\varpi(\cdot)$, which means the epidemic will be persistent long-term. This is supported by the left column of Fig. 1. Furthermore, by detailed calculation, we derive that

$$
\begin{aligned}
& w_{1}=0.0489 \neq 0, \quad w_{2}=7.1555 \neq 0, \\
& w_{3}=-129.2314 \neq 0 .
\end{aligned}
$$

It follows from Theorem 4.1 that the stationary distribution $\varpi(\cdot)$ obeys a log-normal density function $\Phi(S, I, Q, R)$. More precisely,

$$
\begin{aligned}
\Sigma= & \rho_{1}^{2}\left(M_{1} H_{1}\right)^{-1} \Sigma_{0}\left[\left(M_{1} H_{1}\right)^{-1}\right]^{\tau} \\
& +\rho_{2}^{2}\left(M_{2} H_{2} P_{2} J_{2}\right)^{-1} \Sigma_{0}\left[\left(M_{2} H_{2} P_{2} J_{2}\right)^{-1}\right]^{\tau} \\
& +\rho_{3}^{2}\left(M_{3} J_{3}\right)^{-1} \Sigma_{0}\left[\left(M_{3} J_{3}\right)^{-1}\right]^{\tau} \\
& +\rho_{4}^{2}\left(M_{4} J_{4}\right)^{-1} \Sigma_{0}\left[\left(M_{4} J_{4}\right)^{-1}\right]^{\tau} \\
= & 10^{-3} \times\left(\begin{array}{cccc}
0.0491 & -0.0184 & -0.0090 & 0.0040 \\
-0.0184 & 0.1804 & 0.1578 & 0.1764 \\
-0.0090 & 0.1578 & 0.2296 & 0.1852 \\
0.0040 & 0.1764 & 0.1852 & 0.2278
\end{array}\right),
\end{aligned}
$$

By the definition of $E_{+}^{*}$, we further determine that $\left(S_{+}^{*}, I_{+}^{*}, Q_{+}^{*}, R_{+}^{*}\right)=\left(1.0857 \times 10^{5}, 2.8503 \times 10^{5}\right.$, $\left.3.2708 \times 10^{5}, 4.0133 \times 10^{5}\right)$. Then the expression of $\Phi(S, I, Q, R)$ is obtained. In order to validate the correctness of the result, the corresponding marginal density functions of $S(t), I(t), Q(t)$ and $R(t)$ are separately given by
(i) $F_{1}(S)=\frac{\partial \Phi}{\partial S}=56.934 e^{-10183(\ln S-11.6)}$;
(ii) $F_{2}(I)=\frac{\partial \Phi}{\partial I}=29.702 e^{-2771.6(\ln I-12.56)}$;

(iii) $F_{3}(Q)=\frac{\partial \Phi}{\partial Q}=26.328 e^{-2177.7(\ln Q-12.7)}$;

(iv) $F_{4}(R)=\frac{\partial \Phi}{\partial R}=26.432 e^{-2194.9(\ln R-12.9)}$.

The curves of (i)-(iv) are shown in the right column of Fig. 1. Clearly, it verifies Theorem 4.1 from the side.

5.2 Impact of random noises $\sigma_{1}$ and $\sigma_{2}$ on the disease persistence

It follows from Remark 4.1 that random fluctuations of susceptible and infected individuals (i.e., $\sigma_{1}, \sigma_{2}$ ) have a critical influence on the disease persistence. Therefore, by the method of controlling variables, we are devoted to studying the corresponding dynamical effects of $\sigma_{1}$ and $\sigma_{2}$ in the following Example 5.2.

Example 5.2 First, the parameters are chosen considering the following three subcases of random perturbations:

$$
\begin{aligned}
& \left(a_{1}\right)\left(\sigma_{1}, \sigma_{2}\right)=(0.01,0.01) ; \\
& \left(a_{2}\right)\left(\sigma_{1}, \sigma_{2}\right)=(0.1,0.01) ; \\
& \left(a_{3}\right)\left(\sigma_{1}, \sigma_{2}\right)=(0.01,0.1)
\end{aligned}
$$

In fact, the above three subconditions $\left(a_{1}\right)-\left(a_{3}\right)$ all guarantee the existence of the ergodic stationary distribution of system (2.2). For subcases $\left(a_{1}\right)$ and $\left(a_{2}\right)$, i.e., subfigure (2-1), by only increasing the perturbation intensity of susceptible individuals, the corresponding numbers of quarantined and recovered individuals decrease apparently. In contrast, by only increasing the perturbation intensity of infected individuals, the population 

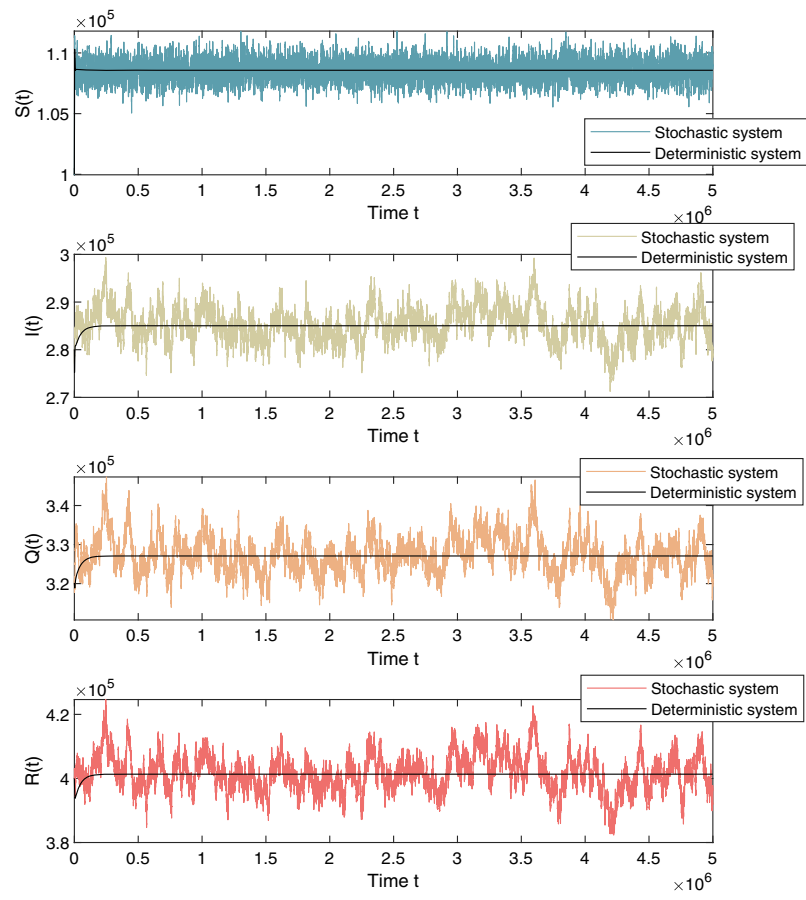

Fig. 1 The left column represents the numbers of $S(t), I(t), Q(t), R(t)$ in model (2.1) and system (2.2) with the initial value $Z(0)$ and the noise intensities $\left(\sigma_{1}, \sigma_{2}, \sigma_{3}, \sigma_{4}\right)=(0.008,0.004,0.005,0.005)$, respectively.

numbers of the quarantined individuals increase apparently, which can be verified by subfigure (2-2). Taken together, both the large white noises $\sigma_{1}$ and $\sigma_{2}$ have a great destabilizing influence on the susceptible and infected population. Figure 2 confirms this.

From the expression of $\mathscr{R}_{0}^{S}$ (or $\mathscr{R}_{0}$ ), the disease persistence of system (2.2) (or system (2.1)) is critically affected by the recruitment rate $\Lambda$, transmission rate $\beta$ and quarantined coefficient $\delta$. Hence, the following Examples 5.3-5.5 will reveal these effects.

\subsection{Impact of recruitment rate $\Lambda$ on the dynamics of system (2.2)}

Example 5.3 Let the stochastic perturbations $\left(\sigma_{1}, \sigma_{2}\right.$, $\left.\sigma_{3}, \sigma_{4}\right)=(0.1,0.1,0.01,0.01)$ and the biological parameters $\left(\beta, \mu, \alpha_{1}, \alpha_{2}, \delta, \gamma, \varepsilon, \omega\right)=\left(5 \times 10^{-7}\right.$, $0.0143,0.02,0.01,0.2,0.2,0.15,0.2)$. Considering the subcases of $\Lambda=10,000,20,000,30,000$ and 40,000, the corresponding population intensities of infected and
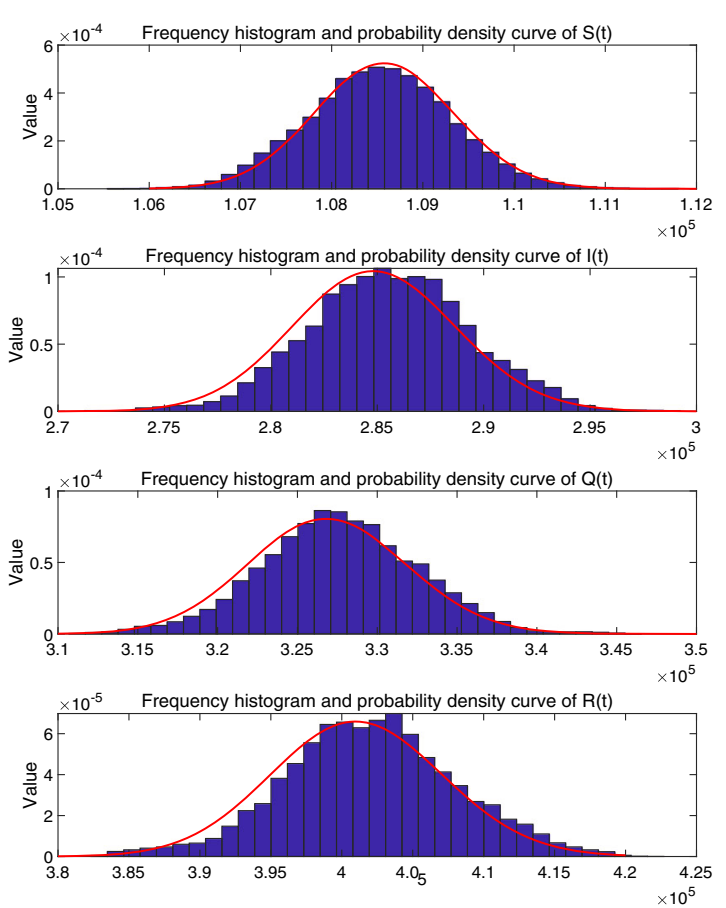

The right column shows the frequency histogram and the corresponding marginal density function curves of individuals $S, I, Q, R$

quarantined individuals are shown in Fig. 3, respectively. Clearly, the epidemic infection will decrease as the recruitment rate decreases. Moreover, a small recruitment rate, such as $\Lambda<10,000$, can effectively lead to disease extinction (see Fig. 3).

5.4 Impact of transmission rate $\beta$ on the dynamics of system (2.2)

Example 5.4 Choose the biological parameters $(\Lambda$, $\left.\mu, \alpha_{1}, \alpha_{2}, \delta, \gamma, \varepsilon, \omega\right)=(20,000,0.0143,0.02,0.01,0.2$, $0.2,0.15,0.2)$ and random noises $\left(\sigma_{1}, \sigma_{2}, \sigma_{3}, \sigma_{4}\right)=$ $(0.1,0.1,0.01,0.01)$. Consider the subcases of transmission rate $\beta=3 \times 10^{-7}, 5 \times 10^{-7}, 7 \times 10^{-7}$ and $9 \times 10^{-7}$, the corresponding population numbers of infected and isolated individuals are described in Fig. 4, respectively. Obviously, smaller contact rate can be conducive to the reduction in infection even lead to elimination of disease. For instance, by Fig. 4, we can take some reasonable measures to guarantee the result of $\beta<3 \times 10^{-7}$ to eliminate the disease. 

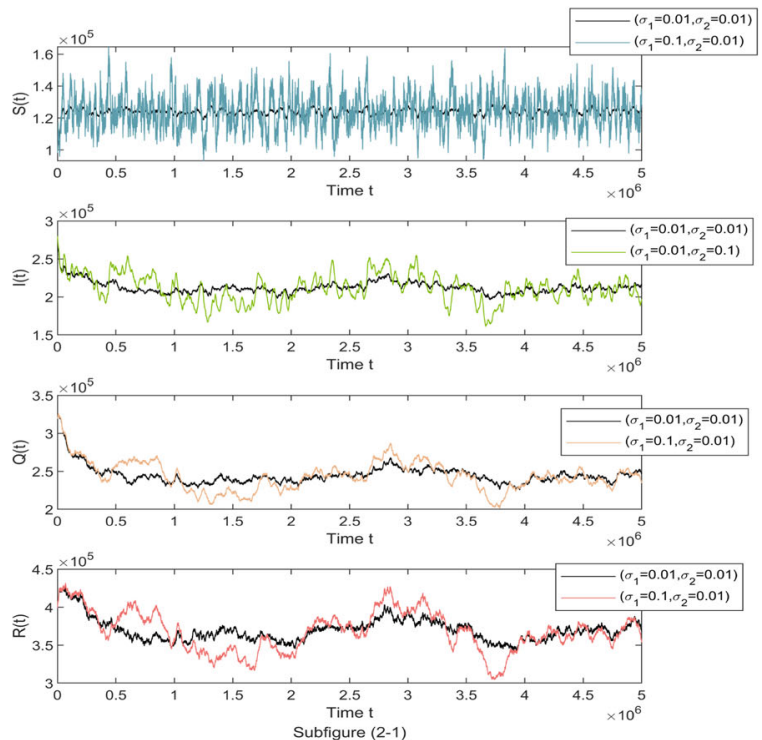

Fig. 2 The corresponding simulations of the solution $\quad(S(t), I(t), Q(t), R(t)) \quad$ to system (2.2) under the noise intensities $\left(\sigma_{1}, \sigma_{2}, \sigma_{3}, \sigma_{4}\right)=$ $(0.01,0.01,0.01,0.01), \quad(0.1,0.01,0.01,0.01) \quad$ and

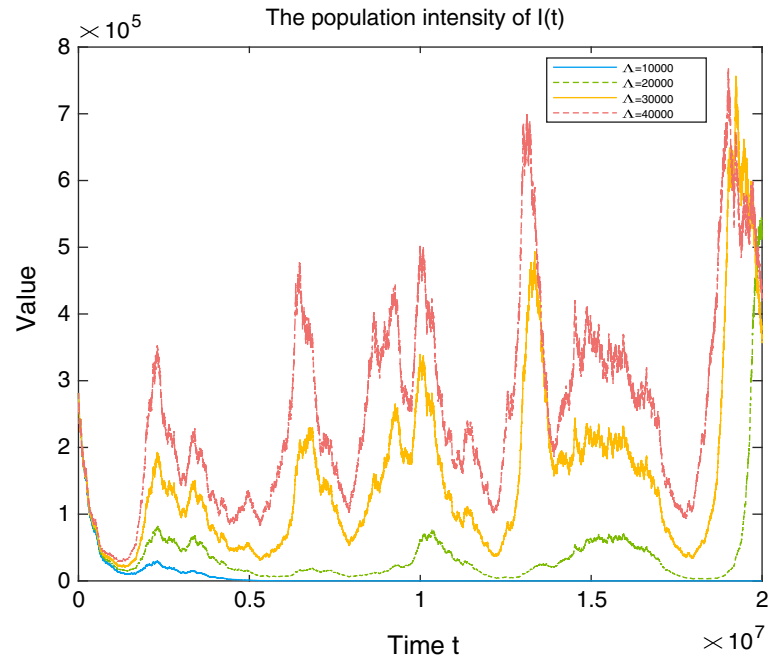

Fig. 3 The population intensities of infected and isolated individuals of system (2.2) with the recruitment rate $\Lambda=10,000,20,000,30,000$ and 40,000,

\subsection{Impact of quarantine coefficient $\delta$ on the dynamics of system (2.2)}

Example 5.5 Consider the environmental fluctuations $\left(\sigma_{1}, \sigma_{2}, \sigma_{3}, \sigma_{4}\right)=(0.1,0.1,0.01,0.01)$ and the bio-
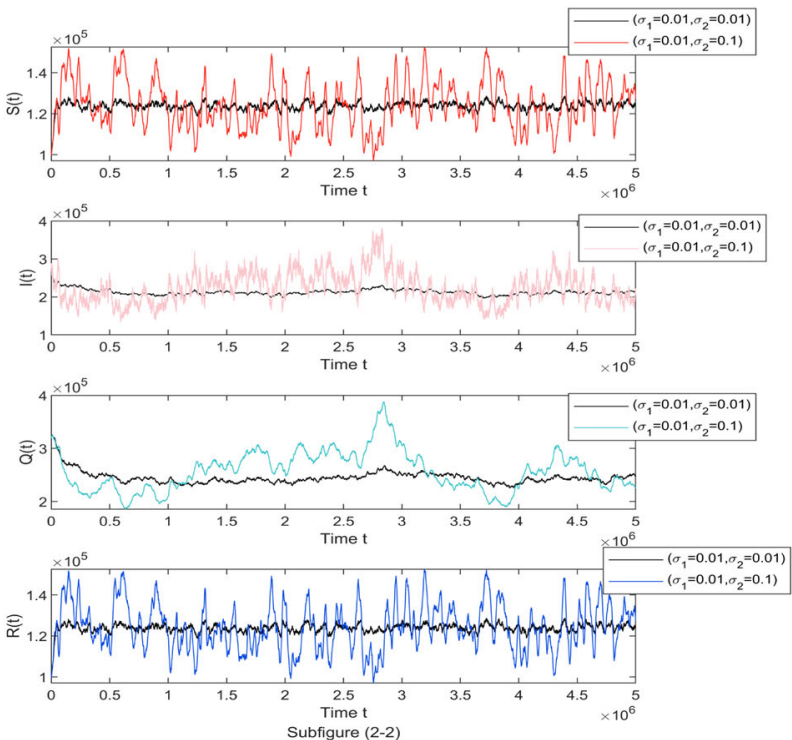

$(0.01,0.1,0.01,0.01)$ are carried out. Other given parameters are as follows: $\left(\Lambda, \beta, \mu, \alpha_{1}, \alpha_{2}, \delta, \gamma, \varepsilon, \omega\right)=$ $\left(20,000,3.5 \times 10^{-6}, 0.0143,0.02,0.01,0.2,0.2,0.15,0.2\right)$ and $\left(\sigma_{3}, \sigma_{4}\right)=(0.01,0.01)$

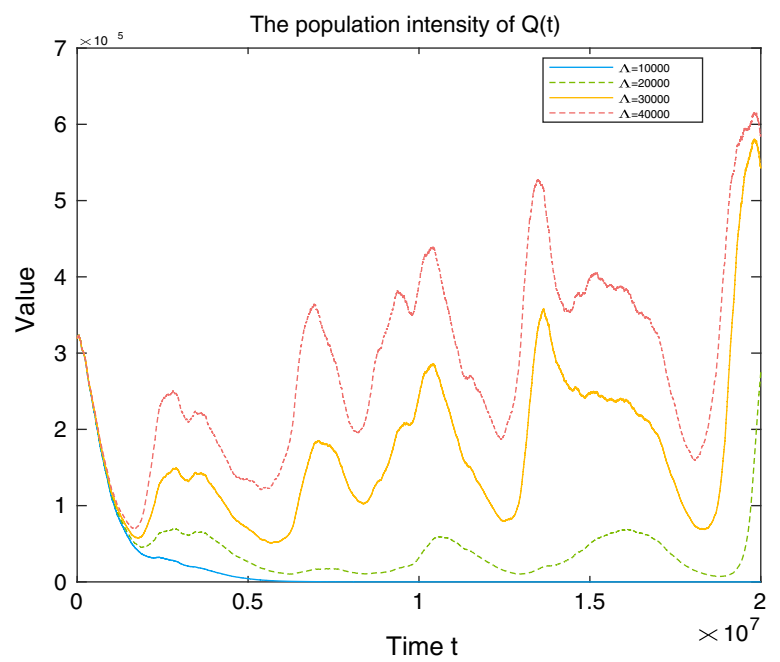

respectively. Other fixed parameters: $\left(\sigma_{1}, \sigma_{2}, \sigma_{3}, \sigma_{4}\right)=$ $(0.1,0.1,0.01,0.01)$ and $\left(\beta, \mu, \alpha_{1}, \alpha_{2}, \delta, \gamma, \varepsilon, \omega\right)=$ $\left(5 \times 10^{-7}, 0.0143,0.02,0.01,0.2,0.2,0.15,0.2\right)$

logical parameters $\left(\Lambda, \beta, \mu, \alpha_{1}, \alpha_{2}, \delta, \gamma, \varepsilon, \omega\right)$ $=\left(20,000,5 \times 10^{-7}, 0.0143,0.02,0.01,0.2,0.15,0.2\right)$. Next, we assume that the quarantined coefficient is $\delta=0.05,0.1,0.2$ and 0.4. Similarly, the disease 


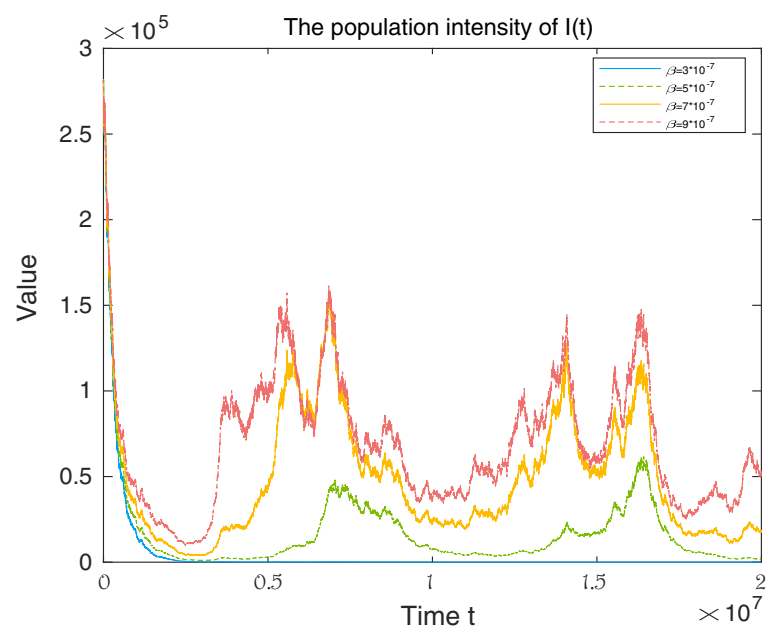

Fig. 4 The population numbers of infected and quarantined individuals of system (2.2) with the transmission rate $\beta=3 \times 10^{-7}, 5 \times 10^{-7}, 7 \times 10^{-7}$ and $9 \times 10^{-7}$, respec-

infection will be under control as the quarantined rate increases. Figure 5 validates this.

For a comprehensive analysis, the case $\mathcal{R}_{0}^{S} \leq 1$ should be discussed and simulated.

5.6 Dynamical behaviors of system (2.2) if $\mathscr{R}_{0}^{S} \leq 1$

Example 5.6 Let the main parameters $\left(\Lambda, \beta, \mu, \alpha_{1}, \alpha_{2}\right.$, $\delta, \gamma, \varepsilon, \omega)=\left(20,000,3.5 \times 10^{-7}, 0.0143,0.02\right.$, $0.01,0.2,0.2,0.15,0.2)$ and the environmental noise intensities $\left(\sigma_{1}, \sigma_{2}, \sigma_{3}, \sigma_{4}\right)=(0.1,0.1,0.01,0.01)$. Then we can compute

$$
\begin{aligned}
& \mathscr{R}_{0}=\frac{\Lambda \beta}{\mu\left(\mu+\alpha_{1}+\delta+\gamma\right)}=1.1283>1, \\
& \mathscr{R}_{0}^{S}=\frac{\Lambda \beta}{\left(\mu+\frac{\sigma_{1}^{2}}{2}\right)\left(\mu+\alpha_{1}+\gamma+\delta+\frac{\sigma_{2}^{2}}{2}\right)}=0.8263<1 .
\end{aligned}
$$

According to Theorem 4.1, the existence and uniqueness of the ergodic stationary distribution of system (2.2) is unknown. Figure 3 indicates that the disease will go to extinction in the long-term. Furthermore, when $\mathscr{R}_{0}>1$, the deterministic system (2.1) has an endemic equilibrium $E^{*}$ which is globally asymptotically stable (Fig. 6).

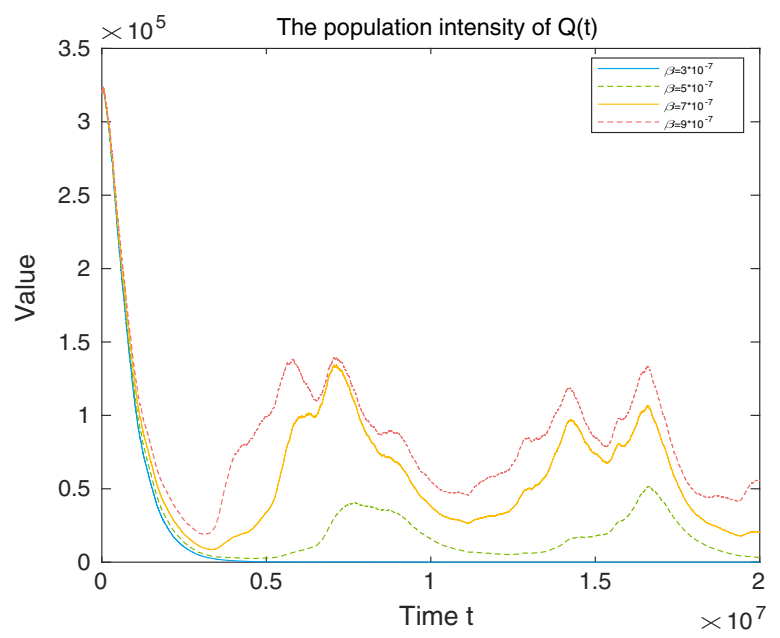

tively. Other given parameters: $\left(\Lambda, \mu, \alpha_{1}, \alpha_{2}, \delta, \gamma, \varepsilon, \omega\right)=$ $(20,000,0.0143,0.02, \quad 0.01,0.2,0.2,0.15,0.2) \quad$ and $\left(\sigma_{1}, \sigma_{2}, \sigma_{3}, \sigma_{4}\right)=(0.1,0.1,0.01,0.01)$

\section{Conclusions and discussion of results}

\subsection{Conclusions}

In this subsection, we draw conclusion from the theoretical results of this paper.

- By means of Theorem 3.1, the existence of the ergodic stationary distribution $\varpi(\cdot)$ of the solution $(S(t), I(t), Q(t), R(t))$ to system (2.2) is proved under $\mathscr{R}_{0}^{S}=\frac{\Lambda \beta}{\left(\mu+\frac{\sigma_{1}^{2}}{2}\right)\left(\mu+\alpha_{1}+\gamma+\delta+\frac{\sigma_{2}^{2}}{2}\right)}>1$.

- By taking the effect of stochasticity into account, the quasi-endemic equilibrium $E_{+}^{*}$ related to $E^{*}$ is defined. Assuming that $\mathscr{R}_{0}^{S}>1$, we determine that the stationary distribution $\varpi(\cdot)$ around $E_{+}^{*}$ admits a log-normal density function in the following form:

$$
\begin{aligned}
& \Phi(S, I, Q, R)=(2 \pi)^{-2}
\end{aligned}
$$

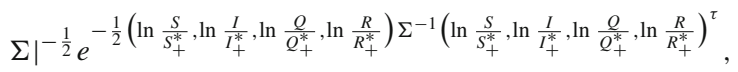

where the special form of $\Sigma$ is shown in Theorem 4.1. Clearly, the above results indicate that the infectious disease will prevail and persist for long-term development if $\mathscr{R}_{0}^{S}>1$. In epidemiology, the first concern is whether an epidemic will occur. Therefore, by means of the above numerical simulations and parameter analyses, we are devoted to providing some effective measures to reduce the threat of infectious diseases to 


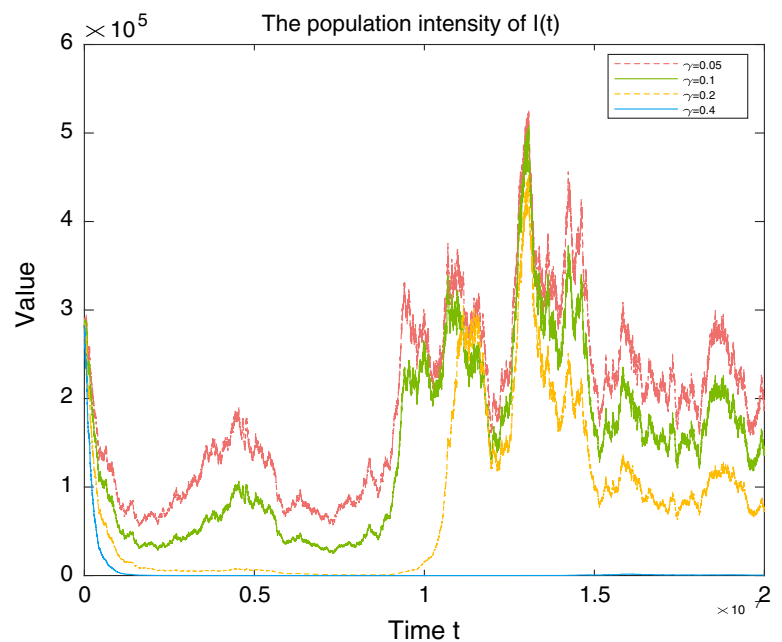

Fig. 5 The numbers of infected and quarantined individuals of system (2.2) with the isolated coefficients $\delta=0.05,0.1,0.2$ and 0.4. Other fixed parameters are as follows: $\left(\sigma_{1}, \sigma_{2}, \sigma_{3}, \sigma_{4}\right)=$

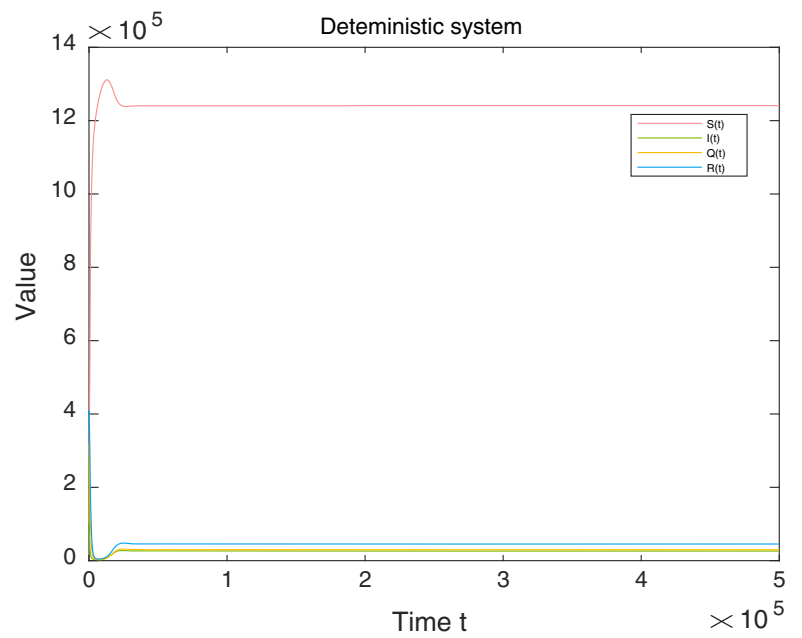

Fig. 6 The left figure shows the numbers of $S(t), I(t), Q(t), R(t) \quad$ in the deterministic system (2.1) with the initial value $Z(0)$. The right figure reflects the population intensities of $S(t), I(t), Q(t), R(t)$ in system

human life and safety, and eventually lead to the eradication of disease. Based on the above numerical simulations, we can conclude the following three points:

(1) To provide effective treatment and a wide range of isolation measures, see Fig. 5.

(2) To control the activities of the susceptible individuals in highly pathogenic areas and provide some

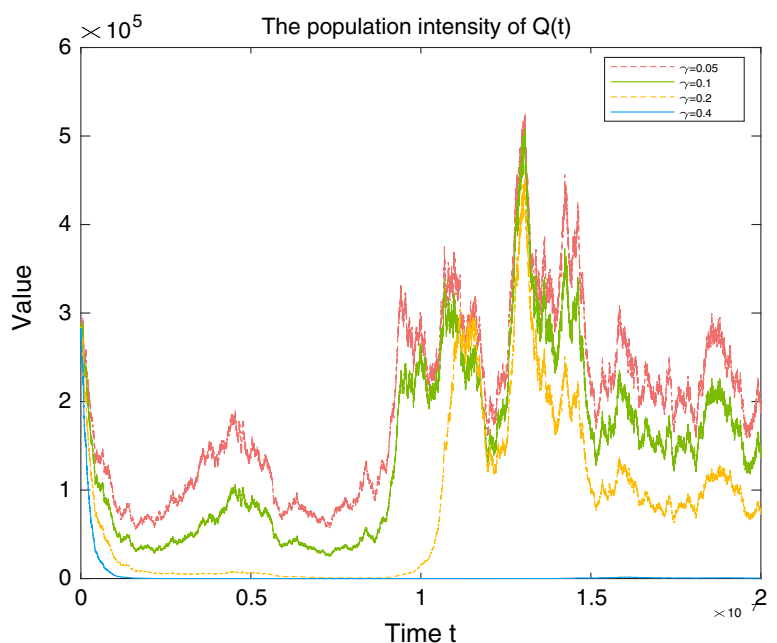

$(0.1,0.1,0.01,0.01)$ and $\left(\Lambda, \beta, \mu, \alpha_{1}, \alpha_{2}, \delta, \gamma, \varepsilon, \omega\right)=$ $\left(20,000,5 \times 10^{-7}, 0.0143,0.02,0.01,0.2,0.15,0.2\right)$

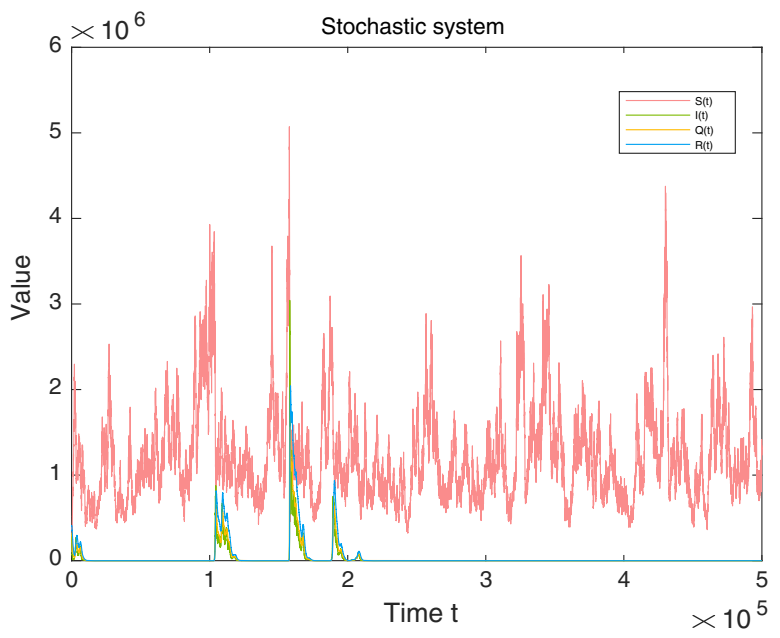

(2.2) with main parameters $\left(\Lambda, \beta, \mu, \alpha_{1}, \alpha_{2}, \delta, \gamma, \varepsilon, \omega\right)=$ $\left(20,000,3.5 \times 10^{-7}, 0.0143,0.02, \quad 0.01,0.2,0.2,0.15,0.2\right)$ and noise intensities $\left(\sigma_{1}, \sigma_{2}, \sigma_{3}, \sigma_{4}\right)=(0.1,0.1,0.01,0.01)$

effective vaccination strategies for susceptible individuals, that is to say, $\beta \rightarrow 0^{+}$, see Fig. 4 .

(3) To implement some reasonable policies to reduce population mobility in differential risk epidemic regions, which means the value of $\Lambda$ is sufficiently small, see Fig. 3. For example, various joint prevention and control greatly inhibited the spread of COVID-19 in China. 
6.2 Discussion of results

In this paper, to the best of our knowledge, the disease persistence of an SIQRS epidemic model, which includes the existence of ergodic stationary distribution and the exact expression of probability density function, is studied. Through comparison with the existing results, our main contributions will be introduced in the following two aspects in detail.

(i) By means of the linear random disturbance shown in [15,20-22,25-37], we focus on a stochastic SIQRS epidemic model with temporary immunity in the present study. Next, we construct some suitable Lyapunov functions to derive a stochastic critical value $\mathscr{R}_{0}^{S}$. By the Khas'minskii ergodicity theory, we obtain that system (2.2) admits a unique ergodic stationary distribution while $\mathscr{R}_{0}^{S}>1$. From the similar expressions of $\mathscr{R}_{0}^{S}$ and the basic reproduction number $\mathscr{R}_{0}$, it greatly reveals that the stochastic positive equilibrium state (i.e., disease persistence) is only determined by the dynamical behavior of the susceptible individuals and the infectious people. More precisely, the corresponding random fluctuations are $\sigma_{1}, \sigma_{2}$. In view of the method of controlling variables and numerical simulations, the key measure to the prevention of infectious disease lies in the control and quarantine of infected individuals. Furthermore, by the corresponding parameter analyses, we further provide some reasonable measures to reduce the transmission of epidemic.

(ii) The existence of the ergodic stationary distribution makes it difficult to determine the exact statistical properties of disease persistence. For further dynamical investigation in epidemiology, based on Zhou and Zhang [33], we develop some solving theories of algebraic equations with respect to the four-dimensional probability density function, which are described in Lemmas 2.3-2.5. In fact, focusing on the previous studies [27-32], the corresponding persistence is only obtained by the existence theory of the unique stationary distribution with ergodicity. By taking the effect of stochasticity into consideration, the quasi-endemic equilibrium $E_{+}^{*}$ is constructed. By means of the equivalence of system (4.1) and the corresponding linearized system (4.3), we derive the exact expression of the log-normal four-dimensional density function $\Phi(S, I, Q, R)$. In addition, the covariance matrix $\Sigma$ is solved by the algebraic equation $G^{2}+A \Sigma+\Sigma A^{\tau}=0$, that is, Eq. (4.6). Following the existing results, the corresponding stability theory of zero solution of the general linear equation, described in [42], can validate the positive definiteness of $\Sigma$. But the specific form of $\Sigma$ is hard to obtain. In the current study, we develop the corresponding standard $R_{1}, R_{2}, R_{3}$ matrices shown in Lemmas 2.3-2.5. By means of the general solving theories, we can verify that $\Sigma$ is positive definite and obtain the special form of $\Sigma$ as shown in the detailed discussions. Furthermore, compared to what the existing results cannot obtain the general expression of $\Sigma$, it is important to highlight that our methods and theories can be used to prove that $\Sigma$ is positive definite even if the diffusion matrix $G$ is semi-positive definite, such as in delay stochastic differential equations [29,43-45].

Finally, some important topics that should be further studied are noted here. First, due to the limitation of our mathematical approaches to an epidemic model with temporary immunity, the sufficient conditions for disease extinction are difficult to establish. Consequently, for a comprehensive discussion, we only plot the relevant simulation of the solution $(S(t), I(t), Q(t), R(t))$ while $\mathscr{R}_{0}^{S} \leq 1$. Second, by taking the effect of telegraph noises into account $[31,35,46]$, the corresponding SIQRS epidemic model with temporary immunity and regime switching should be studied. These problems are expected to be considered and solved in our future work.

Acknowledgements This work is supported by the National Natural Science Foundation of China (No. 11871473) and Shandong Provincial Natural Science Foundation (Nos. ZR2019MA010, ZR2019MA006).

\section{Compliance with ethical standards}

Conflict of interest The authors declare that they have no conflict of interest.

\section{Appendix A}

(I)Proof of Lemma 2.3: Consider the algebraic equation $G_{0}^{2}+A_{0} \theta_{0}+\theta_{0} A_{0}^{\tau}=0$, where $\theta_{0}$ is a symmetric matrix. By direct calculation, we have

$\theta_{0}=\left(\begin{array}{cccc}\sigma_{11} & 0 & \sigma_{13} & 0 \\ 0 & \sigma_{22} & 0 & \sigma_{24} \\ \sigma_{13} & 0 & \sigma_{33} & 0 \\ 0 & \sigma_{24} & 0 & \sigma_{44}\end{array}\right)$, 
where $\sigma_{22}=\frac{a_{3}}{2\left[a_{1}\left(a_{2} a_{3}-a_{1} a_{4}\right)-a_{3}^{2}\right]}, \sigma_{13}=-\sigma_{22}, \sigma_{33}=$ $\frac{a_{1}}{a_{3}} \sigma_{22}, \sigma_{24}=-\frac{a_{1}}{a_{3}}, \sigma_{11}=\frac{a_{2} a_{3}-a_{1} a_{4}}{a_{3}} \sigma_{22}$, and $\sigma_{44}=$ $\frac{a_{1} a_{2}-a_{3}}{a_{3} a_{4}} \sigma_{22}$. Assume that $a_{1}>0, a_{3}>0, a_{4}>$ $0, a_{1}\left(a_{2} a_{3}-a_{1} a_{4}\right)-a_{3}^{2}>0$. Then we can show that $\sigma_{11}>0, \sigma_{11} \sigma_{22}>0, \sigma_{22}\left(\sigma_{11} \sigma_{33}-\sigma_{13}^{2}\right)>0$, $\left(\sigma_{11} \sigma_{33}-\sigma_{13}^{2}\right)\left(\sigma_{22} \sigma_{44}-\sigma_{24}^{2}\right)>0$.

This means all the leading principal minors of matrix $\theta_{0}$ are positive. Consequently, $\theta_{0}$ is positive definite.

The proof is completed.

(II) Proof of Lemma 2.4: Consider the algebraic equation $G_{0}^{2}+B_{0} \theta_{1}+\theta_{1} B_{0}^{\tau}=0$, where $\theta_{1}$ is a symmetric matrix. We can get by direct computation that

$\theta_{1}=\left(\begin{array}{cccc}\theta_{11} & 0 & \theta_{13} & 0 \\ 0 & \theta_{22} & 0 & 0 \\ \theta_{13} & 0 & \theta_{33} & 0 \\ 0 & 0 & 0 & 0\end{array}\right)$

where

$$
\begin{aligned}
& \theta_{22}=\frac{1}{2\left(b_{1} b_{2}-b_{3}\right)}, \theta_{13}=-\theta_{22}, \theta_{11}=b_{2} \theta_{22}, \\
& \theta_{33}=\frac{b_{1}}{b_{3}} \theta_{22} .
\end{aligned}
$$

If $b_{1}>0, b_{3}>0, b_{1} b_{2}-b_{3}>0$, noting that $\theta_{11}>0, \theta_{11} \theta_{22}>0, \theta_{22}\left(\theta_{11} \theta_{33}-\theta_{13}^{2}\right)>0$,

which means three leading principal minors of matrix $\theta_{1}$ are positive. Hence, $\theta_{1}$ is semi-positive definite. The proof is confirmed.

(III) Proof of Lemma 2.5: For the algebraic equation $G_{0}^{2}+C_{0} \theta_{2}+\theta_{2} C_{0}^{\tau}=0$, since $\theta_{2}$ is a symmetric matrix, we obtain

$\theta_{2}=\left(\begin{array}{cccc}\vartheta_{11} & 0 & 0 & 0 \\ 0 & \vartheta_{22} & 0 & 0 \\ 0 & 0 & 0 & 0 \\ 0 & 0 & 0 & 0\end{array}\right)$

where $\vartheta_{11}=\frac{1}{2 c_{1}}, \vartheta_{22}=\frac{1}{2 c_{1} c_{2}}$.

If $c_{1}>0$ and $c_{2}>0$, then $\theta_{2}$ is a semi-positive definite matrix. This completes the proof.

\section{Appendix B (Theory in obtaining standardized transformation matrix)}

By means of the invertible linear transformations, we will derive the corresponding standardized transformation matrices of standard $R_{1}, R_{2}$, and $R_{3}$ matrices.
(I) The theory of obtaining standard $R_{1}$ matrix: For the algebraic equation $G^{2}+A \Sigma+\Sigma A^{\tau}=0$, where $G=\operatorname{diag}(\sigma, 0,0,0)$, and

$A=\left(\begin{array}{cccc}a_{11} & a_{12} & a_{13} & a_{14} \\ a_{21} & a_{22} & a_{23} & a_{24} \\ 0 & a_{32} & a_{33} & a_{34} \\ 0 & 0 & a_{43} & a_{44}\end{array}\right)$

First, we assume that

$a_{21} \neq 0, a_{32} \neq 0, a_{43} \neq 0$.

Define $X=\left(x_{1}, x_{2}, x_{3}, x_{4}\right)^{\tau}$ which follows $d X=$ $A X d t$. Considering the following vector $Y=\left(y_{1}\right.$, $\left.y_{2}, y_{3}, y_{4}\right)^{\tau}$,

$$
\begin{aligned}
y_{4}= & x_{4}, y_{3}=y_{4}^{\prime}=a_{43} x_{3}+a_{44} x_{4}, \\
y_{2}= & y_{3}^{\prime}=a_{43} d x_{3}+a_{44} d x_{4}=a_{32} a_{43} x_{2}+\left(a_{33}\right. \\
& \left.+a_{44}\right) a_{43} x_{3}+\left(a_{44}^{2}+a_{34} a_{43}\right) x_{4}, \\
y_{1}= & y_{2}^{\prime}=a_{21} a_{32} a_{43} x_{1}+\left[\left(a_{22}\right.\right. \\
& \left.\left.+a_{33}+a_{44}\right) a_{32} a_{43}\right] x_{2} \\
& +\left[a _ { 4 3 } \left(a_{23} a_{32}+a_{34} a_{43}\right.\right. \\
& \left.\left.+a_{33} a_{44}+a_{33}^{2}+a_{44}^{2}\right)\right] x_{3} \\
& +\left[a_{24} a_{32} a_{43}+\left(a_{33}+a_{44}\right) a_{34} a_{43}\right. \\
& \left.+\left(a_{34} a_{43}+a_{44}^{2}\right) a_{44}\right] x_{4}:=m_{1} x_{1} \\
& +m_{2} x_{2}+m_{3} x_{3}+m_{4} x_{4} .
\end{aligned}
$$

Then the corresponding standardized transformation matrix is given by

$M=\left(\begin{array}{cccc}m_{1} & m_{2} & m_{3} & m_{4} \\ 0 & a_{32} a_{43} & \left(a_{33}+a_{44}\right) a_{43} & a_{44}^{2}+a_{34} a_{43} \\ 0 & 0 & a_{43} & a_{44} \\ 0 & 0 & 0 & 1\end{array}\right)$

Given the above, we derive that $Y=M X$, which implies that $d Y=M d X=M A X d t=\left(M A M^{-1}\right) Y d t$. Meanwhile, based on the relationship of the vector $Y$ 's components, one has

$d Y=d\left(\begin{array}{l}y_{1} \\ y_{2} \\ y_{3} \\ y_{4}\end{array}\right)=\left(\begin{array}{cccc}-a_{1} & -a_{2} & -a_{3} & -a_{4} \\ 1 & 0 & 0 & 0 \\ 0 & 1 & 0 & 0 \\ 0 & 0 & 1 & 0\end{array}\right)\left(\begin{array}{l}y_{1} \\ y_{2} \\ y_{3} \\ y_{4}\end{array}\right) d t$ 
Obviously, we obtain the corresponding standard $R_{1}$ matrix $M A M^{-1}:=A_{0}$, which refers to (2.3). Let $\rho_{1}=a_{21} a_{32} a_{43} \sigma$ and $\theta_{0}=\rho_{1}^{-2} M \Sigma M^{\tau}$. Then the above equation can be equivalently transformed into the following equation:

$G_{0}^{2}+A_{0} \theta_{0}+\theta_{0} A_{0}^{\tau}=0$.

(II) The method of transforming standard $R_{2}$ matrix: For the algebraic equation $G^{2}+B \Sigma+\Sigma B^{\tau}=0$, where $G=\operatorname{diag}(\sigma, 0,0,0)$, and

$$
B=\left(\begin{array}{cccc}
b_{11} & b_{12} & b_{13} & b_{14} \\
b_{21} & b_{22} & b_{23} & b_{24} \\
0 & b_{32} & b_{33} & b_{34} \\
0 & 0 & 0 & b_{44}
\end{array}\right)
$$

Similarly, we stipulate that

$$
b_{21} \neq 0, b_{32} \neq 0 \text {. }
$$

Let the vector $X=\left(x_{1}, x_{2}, x_{3}, x_{4}\right)^{\tau}$ follow $d X=$ $B X d t$. For the following vector $Y=\left(y_{1}, y_{2}, y_{3}, y_{4}\right)^{\tau}$,

$$
\begin{aligned}
y_{4} & =x_{4}, \quad y_{3}=x_{3}, \quad y_{2}=y_{3}^{\prime}=b_{32} x_{2}+b_{33} x_{3}+b_{34} x_{4}, \\
y_{1} & =y_{2}^{\prime}=b_{32} d x_{2}+b_{33} d x_{3}+b_{34} d x_{4} \\
& =b_{21} b_{32} x_{1}+\left(b_{22}+b_{33}\right) b_{32} x_{2} \\
& +\left(b_{33}^{2}+b_{23} b_{32}\right) x_{3}+\left[\left(b_{33}+b_{44}\right) b_{34}+b_{24} b_{32}\right] x_{4} .
\end{aligned}
$$

Then the relevant standardized transformation matrix $M$ is described by

$$
M=\left(\begin{array}{cccc}
c_{21}\left(c_{11}+c_{22}\right) & c_{12} c_{21}+c_{22}^{2} & c_{13} c_{21}+c_{23}\left(c_{22}+c_{33}\right)+c_{24} c_{43} & c_{14} c_{21}+c_{24}\left(c_{22}+c_{44}\right)+c_{23} c_{34} \\
c_{21} & c_{22} & c_{23} & c_{24} \\
0 & 0 & 1 & 0 \\
0 & 0 & 0 & 1
\end{array}\right),
$$

according to the relationship of the vector $Y$ 's components, we obtain a standard $R_{2}$ matrix $M B M^{-1}:=B_{0}$, which refers to (2.4). Denote $\rho_{2}=b_{21} b_{32} \sigma, \theta_{1}=$ $\rho_{2}^{-2} M \Sigma M^{\tau}$. Then the above equation is equivalent to

$G_{0}^{2}+B_{0} \theta_{1}+\theta_{1} B_{0}^{\tau}=0$.

(III) The method of transforming standard $R_{3}$ matrix: Consider the algebraic equation $G^{2}+C \Sigma+$ $\Sigma C^{\tau}=0$, where $G=\operatorname{diag}(\sigma, 0,0,0)$, and

$C=\left(\begin{array}{cccc}c_{11} & c_{12} & c_{13} & c_{14} \\ c_{21} & c_{22} & c_{23} & c_{24} \\ 0 & 0 & c_{33} & c_{34} \\ 0 & 0 & c_{43} & c_{44}\end{array}\right)$

First, we assume that $c_{21} \neq 0$. For a vector $X=$ $\left(x_{1}, x_{2}, x_{3}, x_{4}\right)^{\tau}$ determined by $d X=C X d t$, the following vector $Y=\left(y_{1}, y_{2}, y_{3}, y_{4}\right)^{\tau}$ satisfies

$$
\begin{aligned}
y_{4}= & x_{4}, y_{3}=x_{3}, y_{2}=c_{21} x_{1} \\
& +c_{22} x_{2}+c_{23} x_{3}+c_{24} x_{4}, \\
y_{1}= & y_{2}^{\prime}=c_{21}\left(c_{11}+c_{22}\right) x_{1}+\left(c_{12} c_{21}+c_{22}^{2}\right) x_{2} \\
& +\left[c_{13} c_{21}+c_{23}\left(c_{22}+c_{33}\right)+c_{24} c_{43}\right] x_{3} \\
& +\left[c_{14} c_{21}+c_{24}\left(c_{22}+c_{44}\right)+c_{23} c_{34}\right] x_{4} .
\end{aligned}
$$

By defining the corresponding standardized transformation matrix
$M=\left(\begin{array}{cccc}b_{21} b_{32} & \left(b_{22}+b_{33}\right) b_{32} & b_{33}^{2}+b_{23} b_{32} & \left(b_{33}+b_{44}\right) b_{34}+b_{24} b_{32} \\ 0 & b_{32} & b_{33} & b_{34} \\ 0 & 0 & 1 & 0 \\ 0 & 0 & 0 & 1\end{array}\right)$

Moreover, we get that $Y=M X$, which means $d Y=M d X=M B X d t=\left(M B M^{-1}\right) Y d t$. Similarly,

we can obtain $Y=M X$. That is to say, $d Y=M d X=$ $M C X d t=\left(M C M^{-1}\right) Y d t$. Hence, the standard $R_{3}$ matrix $M C M^{-1}:=C_{0}$ is obtained, which refers to (2.5). Let $\rho_{3}=c_{21} \sigma$ and $\theta_{2}=\rho_{3}^{-2} M \Sigma M^{\tau}$. Then it can be equivalently transformed into the following equation:

$G_{0}^{2}+C_{0} \theta_{2}+\theta_{2} C_{0}^{\tau}=0$. 


\section{Appendix C}

Consider the following k-dimensional stochastic differential equation

$d X(t)=f(X(t), t) d t+g(X(t), t) d B(t) \quad$ for $t \geq t_{0}$, with the initial value $X(0)=X_{0} \in \mathbb{R}^{k}$, where $B(t)$ depicts a k-dimensional standard Brownian motion defined in the above complete probability space. The common differential operator $\mathscr{L}$ is described by

$$
\begin{aligned}
\mathscr{L}= & \frac{\partial}{\partial t}+\sum_{i=1}^{k} f_{i}(X(t), t) \frac{\partial}{\partial X_{i}} \\
& +\frac{1}{2} \sum_{i, j=1}^{k}\left[g^{\tau}(X(t), t) g(X(t), t)\right]_{i j} \frac{\partial^{2}}{\partial X_{i} \partial X_{j}} .
\end{aligned}
$$

Let the operator $\mathscr{L}$ act on a function $V \in C^{2,1}\left(\mathbb{R}^{k} \times\right.$ $\left.\left[t_{0}, \infty\right] ; \mathbb{R}_{+}^{1}\right)$. Then one can determine that

$$
\begin{gathered}
\mathscr{L} V(X, t)=V_{t}(X(t), t)+V_{X}(X(t), t) f(X(t), t) \\
+\frac{1}{2} \text { trace }\left[g^{\tau}(X(t), t) V_{X X}(X(t), t) g(X(t), t)\right],
\end{gathered}
$$

where $V_{t}=\frac{\partial V}{\partial t}, V_{X}=\left(\frac{\partial V}{\partial x_{1}}, \ldots, \frac{\partial V}{\partial x_{k}}\right)$, and $V_{X X}=$ $\left(\frac{\partial^{2} V}{\partial x_{i} \partial x_{j}}\right)_{k \times k}$. If $X(t) \in \mathbb{R}^{k}$, we have

$$
\begin{aligned}
& d V(X(t), t)=\mathscr{L} V(X(t), t) d t \\
& \quad+V_{X}(X(t), t) g(X(t), t) d B(t) .
\end{aligned}
$$

\section{References}

1. Khan, T., Zaman, G., Chohan, M.I.: The transmission dynamic of different hepatitis B-infected individuals with the effect of hospitalization. J. Biol. Dyn. 12, 611-631 (2018)

2. Sasaki, S., Suzuki, H., Fujino, Y., Kimura, Y., Cheelo, M.: Impact of drainage networks on cholera outbreaks in Lusaka, Zambia. Am. J. Public Health 99, 1982-1989 (2009)

3. Ma, X., Wang, W.: A discrete model of avian influenza with seasonal reproduction and transmission. J. Biol. Dyn. 4, 296-314 (2010)

4. Kermack, W.O., McKendrick, A.G.: A contribution to the mathematical theory of epidemics. Proc. R. Soc. Lond. A 115, 700-21 (1927)

5. Liu, X., Takeuchi, Y., Iwami, S.: SVIR epidemic models with vaccination strategies. J. Theor. Biol. 253, 1-11 (2008)

6. Li, J., Teng, Z., Wang, G., Zhang, L., Hu, C.: Stability and bifurcation analysis of an SIR epidemic model with logistic growth and saturation treatment. Chaos Soliton Fractals 99, 63-71 (2017)

7. Jerubet, R., Kimathi, G.: Analysis and modeling of tuberculosis transmission dynamics. J. Adv. Math. Comput. Sci. 32, 1-14 (2019)
8. Li, M.Y., Smith, H.L., Wang, L.: Global dynamics of an SEIR epidemic model with vertical transmission. SIAM. J. Appl. Math. 62, 58-69 (2001)

9. Hove-Musekwa, S.D., Nyabadza, F.: The dynamics of an HIV/AIDS model with screened disease carriers. Comput. Math. Method Med. 10, 287-305 (2015)

10. Iwami, S., Takeuchi, Y., Liu, X.: Avian-human influenza epidemic model. Math. Biosci. 207, 1-25 (2007)

11. Cai, L., Wu, J.: Analysis of an HIV/AIDS treatment model with a nonlinear incidence. Chaos Soliton Fractals 41, 175182 (2009)

12. Vincenzo, C., Gabriella, S.: A generalization of the Kermack-McKendrick deterministic epidemic model. Math. Biosci. 42, 43-61 (1978)

13. Carter, E., Currie, C.C., Asuni, A., et al.: The first six weekssetting up a UK urgent dental care centre during the COVID19 pandemic. Br. Dent. J. 228, 842-848 (2020)

14. Liu, J., Zhou, Y.: Global stability of an SIRS epidemic model with transport-related infection. Chaos Soliton Fractals 40, 145-158 (2009)

15. Hethcode, H., Ma, Z., Liao, S.: Effect of quarantine in six endemic models for infectious diseases. Math. Biosci. 180, 141-160 (2002)

16. Ma, Y., Liu, J., Li, H.: Global dynamics of an SIQR model with vaccination and elimination hybrid strategies. Mathematics 6, 328 (2018)

17. Joshi, H., Sharma, R.K., Prajapati, G.L.: Global dynamics of an SIQR epidemic model with saturated incidence rate. Asian J. Math. Comput. Res. 21, 156-166 (2017)

18. Feng, Z., Thieme, H.R.: Recurrent outbreaks of childhood diseases revisited: the impact of isolation. Math. Biosci. 128, 93-130 (1995)

19. Wu, L., Feng, Z.: Homoclinic bifurcation in an SIQR model for childhood diseases. J. Differ. Equ. 168, 150-167 (2000)

20. Zhang, X., Huo, H., Xiang, H., Meng, X.: Dynamics of the deterministic and stochastic SIQS epidemic model with nonlinear incidence. Appl. Math. Comput. 243, 546-558 (2014)

21. Ma, Z., Zhou, Y., Wu, J.: Modeling and Dynamic of Infectious Disease. Higher Education Press, Beijing (2009)

22. Shuai, Z., Tien, J.H., Driessche, P.: Cholera models with hyperinfectivity and temporary immunity. Bull. Math. Biol. 74, 2423-2445 (2012)

23. Li, X., Gray, A., Jiang, D., Mao, X.: Sufficient and necessary conditions of stochastic permanence and extinction for stochastic logistic populations under regime switching. J. Math. Anal. Appl. 376, 11-28 (2011)

24. Liu, Q., Jiang, D., Hayat, T., Ahmad, B.: Analysis of a delayed vaccinated SIR epidemic model with temporary immunity and Lévy jumps. Nonlinear Anal. Real. 27, 29-43 (2018)

25. Cai, Y., Kang, Y.: A stochastic epidemic model incorporating media coverage. Commun. Math. Sci. 14, 893-910 (2015)

26. Zhao, Y., Jiang, D.: The threshold of a stochastic SIS epidemic model with vaccination. Appl. Math. Comput. 243, 718-727 (2014)

27. Khan, T., Khan, A.: The extinction and persistence of the stochastic hepatitis B epidemic model. Chaos Soliton Fractals 108, 123-128 (2018)

28. Han, B., Jiang, D., et al.: Stationary distribution and extinction of a stochastic staged progression AIDS model with 
staged treatment and second-order perturbation. Chaos Soliton Fractals 140, 110238 (2020)

29. Zhang, X.: Global dynamics of a stochastic avian-human influenza epidemic model with logistic growth for avian population. Nonlinear Dyn. 90, 2331-2343 (2017)

30. Caraballo, T., Fatini, M.E., Khalifi, M.E.: Analysis of a stochastic distributed delay epidemic model with relapse and Gamma distribution kernel. Chaos Soliton Fractals 133, 109643 (2020)

31. Wang, Y., Jiang, D.: Stationary distribution of an HIV model with general nonlinear incidence rate and stochastic perturbations. J. Frankl. I(356), 6610-6637 (2019)

32. Wang, L., Wang, K., et al.: Nontrivial periodic solution for a stochastic brucellosis model with application to Xinjiang, China. Physica A 510, 522-537 (2018)

33. Liu, Q., Jiang, D., Hayat, T., Alsaedi, A.: Dynamical behavior of a stochastic epidemic model for cholera. J. Frankl. I(356), 7486-7514 (2019)

34. Zhou, B., Zhang, X., Jiang, D.: Dynamics and density function analysis of a stochastic SVI epidemic model with half saturated incidence rate. Chaos Soliton Fractals 137, 109865 (2020)

35. Qi, K., Jiang, D.: The impact of virus carrier screening and actively seeking treatment on dynamical behavior of a stochastic HIV/AIDS infection model. Appl. Math. Model. 85, 378-404 (2020)

36. Zhang, X., Jiang, D., Alsaedi, A.: Stationary distribution of stochastic SIS epidemic model with vaccination under regime switching. Appl. Math. Lett. 59, 87-93 (2016)

37. Mao, X.: Stochastic Differential Equations and Applications. Horwood Publishing, Chichester (1997)

38. Liu, Q., Jiang, D., Shi, N., Hayat, T., Ahmad, B.: Stationary distribution and extinction of a stochastic SEIR epidemic model with standard incidence. Physica A 476, 58-69 (2017)
39. Has'miniskii, R.Z.: Stochastic Stability of Differential equations. Sijthoff Noordhoff, Alphen aan den Rijn (1980)

40. Gardiner, C.W.: Handbook of Stochastic Methods for Physics. Chemistry and the Natural Sciences. Springer, Berlin (1983)

41. Roozen, H.: An asymptotic solution to a two-dimensional exit problem arising in population dynamics. SIAM J. Appl. Math. 49, 1793 (1989)

42. Higham, D.J.: An algorithmic introduction to numerical simulation of stochastic differential equations. SIAM Rev. 43, 525-546 (2001)

43. Ma, Z., Zhou, Y., Li, C.: Qualitative and Stability Methods for Ordinary Differential Equations. Science Press, Beijing (2015)

44. Liu, Q., Jiang, D., Hayat, T., Alsaedi, A.: Long-time behaviour of a stochastic chemostat model with distributed delay. Stochastics 91, 1141-1163 (2019)

45. Li, M.Y., Shuai, Z., Wang, C.: Global stability of multigroup epidemic models with distributed delays. J. Math. Anal. Appl. 361, 38-47 (2010)

46. Liu, Q., Jiang, D., Shi, N., Hayat, T., Alsaedi, A.: Asymptotic behavior of stochastic multi-group epidemic models with distributed delays. Physica A 467, 527-541 (2017)

47. Liu, Q., Jiang, D., Shi,N., Hayat,T., et al.: A stochastic SIRS epidemic model with logistic growth and general nonlinear incidence rate. Phys A Stat Mech Appl 551, 124152 (2020)

Publisher's Note Springer Nature remains neutral with regard to jurisdictional claims in published maps and institutional affiliations. 